Preprint typeset in JHEP style - HYPER VERSION

arXiv:0707.1651

DESY-07-072

HD-THEP-07-14

JHEP 0709(2007)113

\title{
Local SU(5) Unification from the Heterotic String
}

\author{
W. Buchmüller ${ }^{a}$, C. Lüdeling ${ }^{b}$, J. Schmidt ${ }^{a}$ \\ a Deutsches Elektronen-Synchrotron DESY, Hamburg, Germany \\ $b$ Institut für Theoretische Physik, Universität Heidelberg, Heidelberg, Germany
}

\begin{abstract}
We construct a 6D supergravity theory which emerges as intermediate step in the compactification of the heterotic string to the supersymmetric standard model in four dimensions. The theory has $\mathcal{N}=2$ supersymmetry and a gravitational sector with one tensor and two hypermultiplets in addition to the supergravity multiplet. Compactification to four dimensions occurs on a $T^{2} / \mathbb{Z}_{2}$ orbifold which has two inequivalent pairs of fixed points with unbroken $\mathrm{SU}(5)$ and $\mathrm{SU}(2) \times \mathrm{SU}(4)$ symmetry, respectively. All gauge, gravitational and mixed anomalies are cancelled by the Green-Schwarz mechanism. The model has partial 6D gauge-Higgs unification. Two quark-lepton generations are localized at the SU(5) branes, the third family is composed of split bulk hypermultiplets. The top Yukawa coupling is given by the 6D gauge coupling, all other Yukawa couplings are generated by higher-dimensional operators at the $\mathrm{SU}(5)$ branes. The presence of the $\mathrm{SU}(2) \times \mathrm{SU}(4)$ brane breaks $\mathrm{SU}(5)$ and generates split gauge and Higgs multiplets with $\mathcal{N}=1$ supersymmetry in four dimensions. The third generation is obtained from two split $\overline{\mathbf{5}}$-plets and two split 10-plets, which together have the quantum numbers of one $\overline{\mathbf{5}}$-plet and one $\mathbf{1 0}$ plet. This avoids unsuccessful SU(5) predictions for Yukawa couplings of ordinary $4 \mathrm{D} \mathrm{SU}(5)$ grand unified theories.
\end{abstract}

KEYWords: Superstrings and Heterotic Strings, Superstring Vacua. 


\section{Contents}

1. Introduction 1

2. 6D Supergravity from the Heterotic String 3

2.1 The Heterotic String on $T^{6} / \mathbb{Z}_{6-\mathrm{II}}$

2.2 Intermediate $\mathbb{Z}_{3}$ Compactification

3. $\mathbb{Z}_{2}$ Compactification to Four Dimensions 8

4. Anomalies 14

4.1 Anomalies and the Green-Schwarz Mechanism 15

4.2 Bulk Anomalies 16

4.3 Brane Anomalies 18

5. Decoupling of Exotic States 20

6. Yukawa Couplings 22

7. Supersymmetric Vacua 24

8. Outlook 26

A. States 28

A.1 R-Charges 28

A.2 Bulk States 29

A.3 States at the Fixed Points 31

B. Anomaly Polynomials 37

References 39

\section{Introduction}

The symmetries and the particle content of the standard model point towards grand unified theories (GUTs). The simplest unified gauge group is $\mathrm{SU}(5)$ with three $\overline{\mathbf{5}}$ - and 10-plets for the three quark-lepton generations of the standard model [1]. Higgs doublets can be obtained from further $\mathbf{5}$ - and $\overline{\mathbf{5}}$-plets, with their heavy color triplet partners decoupled from the low energy theory. In supersymmetric GUTs the hierarchy 
between the electroweak scale and the GUT scale is stabilized and, for the minimal case of two Higgs doublets, gauge couplings unify at the scale $M_{\mathrm{GUT}} \simeq 2 \times 10^{16} \mathrm{GeV}$.

Neutrino masses and mixings can be described by adding a non-renormalizable, lepton-number violating dimension-5 operator composed of lepton and Higgs doublets, with coupling strength $1 / \Lambda$. The observed smallness of the neutrino masses then requires $\Lambda=\mathcal{O}\left(M_{\mathrm{GUT}}\right)$, hinting at a $B-L$ breaking scale of the order of $M_{\mathrm{GUT}}$. Embedding $\mathrm{SU}(5)$ and $\mathrm{U}(1)_{B-L}$ in $\mathrm{SO}(10)[2,3]$, and continuing the route of unification via exceptional groups, one arrives at $\mathrm{E}_{8}$, which is beautifully realized in the heterotic string $[4,5]$.

An elegant scheme leading to chiral gauge theories in four dimensions is the compactification on orbifolds [6-10]. Recently, considerable progress has been made in deriving unified field theories from orbifold compactifications of the heterotic string [11-16], and it has been demonstrated that the idea of local grand unification can serve as a guide to find string vacua corresponding to the supersymmetric standard model [17-19]. In this paper we study in some detail an orbifold GUT limit of the model [17], where two of the compact dimensions are larger than the other four. In this way we hope to obtain a better understanding of some open questions of current orbifold compactifications: the large vacuum degeneracy, the decoupling of unwanted massless states and the stabilization of moduli fields.

The model [17] is based on a $\mathbb{Z}_{6-\text { II }}$ twist which is the product of a $\mathbb{Z}_{3}$ twist and a $\mathbb{Z}_{2}$ twist. In a first step, described in Section 2, we compactify the $\mathrm{E}_{8} \times \mathrm{E}_{8}$ heterotic string on the orbifold $T^{4} / \mathbb{Z}_{3}$, where $T^{4}$ is a 4 -torus with the Lie algebra lattice $\mathrm{G}_{2} \times \mathrm{SU}(3)$. The six-dimensional (6D) theory has $\mathcal{N}=2$ supersymmetry and unbroken gauge group

$$
\mathrm{G}_{6}=\mathrm{SU}(6) \times \mathrm{U}(1)^{3} \times\left[\mathrm{SU}(3) \times \mathrm{SO}(8) \times \mathrm{U}(1)^{2}\right],
$$

where the brackets denote the subgroup of the second $\mathrm{E}_{8}$. The gravitational sector contains one tensor multiplet whose (anti-)self-dual part belongs to the $\mathcal{N}=2$ (dilaton) supergravity multiplet.

Compactification from six to four dimensions on the orbifold $T^{2} / \mathbb{Z}_{2}$ with $\mathrm{SO}(4)$ Lie lattice leads to additional fixed points and twisted sectors. The massless spectrum in four dimensions agrees with the results obtained in $[17,18]$. In addition to the zero modes, the 6D field theory contains the Kaluza-Klein excitations of the large $\mathrm{SO}(4)$ plane and further non-Abelian singlets. As described in Section 3, the projection conditions for physical massless states of the model [17] now become $\mathbb{Z}_{2}$ projection conditions for the $6 \mathrm{D}$ bulk fields at the orbifold fixed points in the $\mathrm{SO}(4)$-plane.

Given the $\mathbb{Z}_{2}$ parities of the $6 \mathrm{D}$ bulk fields, one can perform a highly non-trivial consistency check of the 6D field theory, the cancellation of all gauge, gravitational and mixed anomalies by the Green-Schwarz mechanism [20]. In Section 4 it is explicitly shown that all irreducible anomalies vanish and that the reducible ones are indeed cancelled by a unique Green-Schwarz term in the effective action [21,22]. The 
$6 \mathrm{D}$ theory has different local anomalous $\mathrm{U}(1)$ symmetries at the different fixed points in the SO(4) plane. Their sum yields the anomalous $\mathrm{U}(1)$ of the $4 \mathrm{D}$ theory [18].

The 6D theory has a GUT gauge group and $\mathcal{N}=2$ supersymmetry, and therefore considerably fewer multiplets than the 4D theory. This simplifies the decoupling of unwanted exotic states as we show in Section 5. For a vacuum with spontaneously broken $B-L$ symmetry we then obtain a local SU(5) GUT model with two localized and two bulk quark-lepton families. The Higgs fields are identified as bulk fields with partial gauge-Higgs unification. The SU(5) invariant Yukawa couplings and the $\mathrm{SU}(5)$ breaking by the $\mathbb{Z}_{2}$ orbifolding are discussed in Section 6. Open problems concerning supersymmetric vacua and the stabilization of the compact dimensions are outlined in Section 7.

Finally, in Section 8, we conclude with a brief outlook on open questions and further challenges for realistic compactifications of the heterotic string.

\section{6D Supergravity from the Heterotic String}

\subsection{The Heterotic String on $T^{6} / \mathbb{Z}_{6-I I}$}

We consider the propagation of the $\mathrm{E}_{8} \times \mathrm{E}_{8}$ heterotic string in a space-time background which is the product of four-dimensional Minkowski space and a sixdimensional orbifold [23]. The compact space is obtained by dividing the torus $T^{6}=\mathbb{R}^{6} / 2 \pi \Lambda$ by the discrete symmetry $\mathbb{Z}_{6-\mathrm{II}}=\mathbb{Z}_{3} \times \mathbb{Z}_{2}$ of the Lie algebra lattice $\mathrm{SO}(4) \times \mathrm{SU}(3) \times \mathrm{G}_{2}$. The four complex coordinates $z^{i}, i=1 \ldots 4$, comprise the two transverse dimensions of Minkowski space $(i=4)$ and the six compact dimensions $(i=1 \ldots 3)$.

The $\mathbb{Z}_{6-\mathrm{II}}$ orbifold with the $\mathrm{G}_{2} \times \mathrm{SU}(3) \times \mathrm{SO}(4)$ lattice is characterized by the twist vector

$$
v_{6}=\left(-\frac{1}{6},-\frac{1}{3}, \frac{1}{2} ; 0\right),
$$

which is the sum of $\mathbb{Z}_{3}$ and $\mathbb{Z}_{2}$ twist vectors, $v_{6}=-v_{3}+v_{2}$, where

$$
v_{3}=2 v_{6}, \quad v_{2}=3 v_{6}
$$

Note that the $\mathbb{Z}_{3}$ twist leaves the $\mathrm{SO}(4)$ plane invariant whereas the $\mathbb{Z}_{2}$ twist does not affect the $\mathrm{SU}(3)$ plane. Both twists act non-trivially on the $\mathrm{G}_{2}$ plane.

In the light-cone gauge the heterotic string can be described by 4 complex coordinates $Z^{i}(\sigma)(i=1 \ldots 4), 4$ bosonized right-moving Neveu-Schwarz-Ramond (NSR) fermions $H^{i}\left(\sigma_{-}\right)(i=1 \ldots 4)$ and 16 left-moving bosons $X^{I}\left(\sigma_{+}\right)(I=1 \ldots 16)$, where

$\sigma_{ \pm}=\tau \pm \sigma$. The fields $X^{I}$ are compactified on the $16-$ dimensional $\mathrm{E}_{8} \times \mathrm{E}_{8}$ torus. Correspondingly, the momenta of the right-moving fields $H^{i}$ lie on the weight lattice of the little group $\mathrm{SO}(8)$. The quantum numbers of a string state are thus given 
by the $\mathrm{E}_{8} \times \mathrm{E}_{8}$ root vector $p^{I}$ for the gauge and the $\mathrm{SO}(8)$ weight vector $q^{i}$ for the Lorentz quantum numbers.

The orbifold twist is embedded into the gauge group by the $\mathbb{Z}_{6}$ twist vector

$$
V_{6}=\left(-\frac{1}{2},-\frac{1}{2}, \frac{1}{3}, 0^{5}\right)\left(\frac{17}{6},\left(-\frac{5}{2}\right)^{6}, \frac{5}{2},\right) .
$$

In addition, there are two Wilson lines associated with the two subtwists: a $\mathbb{Z}_{3}$ Wilson line $W_{3}$ in the $\mathrm{SU}(3)$ plane and a $\mathbb{Z}_{2}$ Wilson line $W_{2}$ in the $\mathrm{SO}(4)$ plane, given by

$$
\begin{aligned}
& W_{3}=\left(-\frac{1}{6}, \frac{1}{2}, \frac{1}{2},\left(-\frac{1}{6}\right)^{5}\right)\left(0,-\frac{2}{3}, \frac{1}{3}, \frac{4}{3},-1,0^{3}\right), \\
& W_{2}=\left(-\frac{1}{2}, 0,-\frac{1}{2}, \frac{1}{2}, \frac{1}{2}, 0^{3}\right)\left(\frac{23}{4},-\frac{25}{4},-\frac{21}{4},-\frac{19}{4},-\frac{25}{4},-\frac{21}{4},-\frac{17}{4}, \frac{17}{4}\right) .
\end{aligned}
$$

A basis in the Hilbert space of the quantized string is obtained by acting with the creation operators $(n<0)$ for right-handed modes $\left(\alpha_{n}^{i}, \widetilde{\beta}_{n}^{i}\right)$ and left-handed modes $\left(\widetilde{\alpha}_{n}^{i}, \widetilde{\alpha}_{n}^{I}\right)$ on the ground states of the untwisted sector $U(k=0)$ and the twisted sectors $T_{k}(k=1 \ldots 5)$. The ground states of the different sectors depend on the momentum vectors $q^{i}, p^{I}$ and, for the twisted sectors, also on the fixed point $f$ (cf. [18, 23]),

$$
|q, p\rangle \equiv|q\rangle \otimes|p\rangle, \quad|f ; q, p\rangle \equiv\left|q_{\mathrm{sh}}\right\rangle \otimes\left|p_{\mathrm{sh}}\right\rangle
$$

with the shifted momenta

$$
q_{\mathrm{sh}}=q+k v_{6}, \quad p_{\mathrm{sh}}=p+V_{f} .
$$

Here $k$ is the order of the twist and $V_{f}$ is the local gauge twist at the fixed point $f$. It turns out that for the considered model only oscillator modes of the left-moving strings $Z_{\mathrm{L}}^{i}\left(\sigma_{+}\right), Z_{\mathrm{L}}^{* i}\left(\sigma_{+}\right)$and $X^{I}\left(\sigma_{+}\right)$are relevant.

\subsection{Intermediate $\mathbb{Z}_{3}$ Compactification}

We are now interested in the effective field theory for the massless states in the limit where the $\mathrm{SO}(4)$ plane is much larger the $\mathrm{G}_{2}$ and $\mathrm{SU}(3)$ planes, yielding approximately flat 6D Minkowski space. Hence, in a first step, we consider the compactification on the orbifold $T^{4} / \mathbb{Z}_{3}$. The physical states of the gravitational sector,

$$
|q, i\rangle=|q\rangle \otimes \widetilde{\alpha}_{-1}^{i}|0\rangle, \quad\left|q, i^{*}\right\rangle=|q\rangle \otimes \widetilde{\alpha}_{-1}^{* i}|0\rangle,
$$

have to satisfy the mass equations

$$
\frac{1}{8} m_{\mathrm{R}}^{2}=\frac{1}{2} q^{2}-\frac{1}{2}=0,
$$




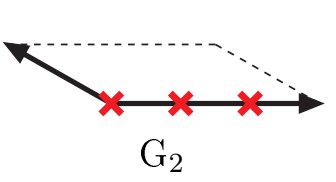

$\mathrm{G}_{2}$

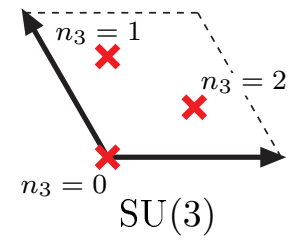

$(0,0)$

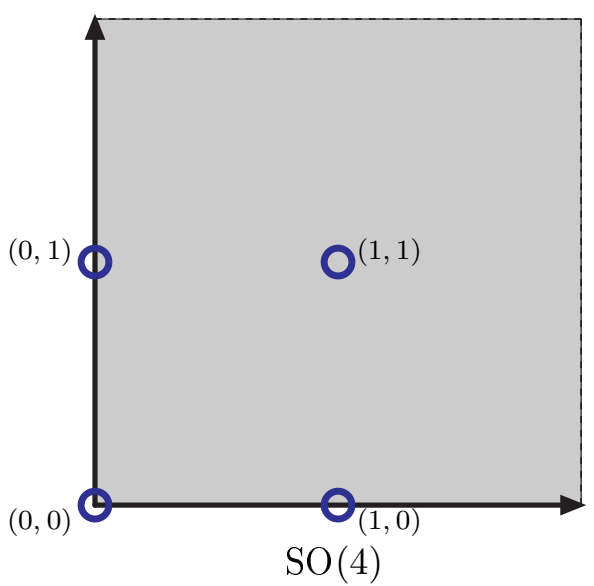

Figure 1: The tori of the orbifold $T^{6} / \mathbb{Z}_{6}$. Red crosses mark fixed points of the $\mathbb{Z}_{3}$ twist used for the first step of compactification. The $S O(4)$ torus is invariant, while the other tori contain three fixed points each. The fixed points in the $G_{2}$ torus are equivalent, while the $S U(3)$ torus contains a Wilson line, and the fixed points are inequivalent and labelled by $n_{3}$. The blue circles mark the $\mathbb{Z}_{2}$ fixed points in the $S O(4)$ plane which are labelled by $\left(n_{2}, n_{2}^{\prime}\right)$. There are further $\mathbb{Z}_{2}$ fixed points in the $G_{2}$ torus which are not shown.

$$
\frac{1}{8} m_{\mathrm{L}}^{2}=\frac{1}{2} p^{2}-1+\widetilde{N}+\widetilde{N}^{*}=0
$$

Here $p=0$, and $\widetilde{N}, \widetilde{N}^{*}$ are the oscillator numbers for left-moving modes in $z^{i}, z^{* i}$ directions, summed over $i$ : $\widetilde{N}=\sum_{i} \widetilde{N}_{i}, \widetilde{N}^{*}=\sum_{i} \widetilde{N}_{i}^{*}$. Furthermore, physical states have to be invariant under the $\mathbb{Z}_{3}$ twist,

$$
v_{3} \cdot\left(\tilde{N}-\tilde{N}^{*}-q\right)=0 \bmod 1
$$

The 16 bosonic states ${ }^{1} q=(0,0, \pm 1,0)$ with $i=3,4$, together with the 16 fermionic states $q=\left(\frac{1}{2}, \frac{1}{2}, \pm \frac{1}{2}, \pm \frac{1}{2}\right),\left(-\frac{1}{2},-\frac{1}{2}, \pm \frac{1}{2}, \pm \frac{1}{2}\right)$ with $i=3,4$, form the familiar $6 \mathrm{D}$ supergravity and dilaton $N=2$ multiplets [24],

$$
\left(G_{M N}, B_{M N}^{+}, \Psi_{M}\right), \quad\left(B_{M N}^{-}, \Phi, \chi\right)
$$

Here $B_{M N}^{+}\left(B_{M N}^{-}\right)$is the antisymmetric tensor field with (anti-)self-dual field strength. Note that together there is only one tensor field $B_{M N}$ without self-duality conditions, which is the special case for which a lagrangian exists.

The 4 bosonic states $q=(1,0,0,0),(0,-1,0,0)$ with $\widetilde{N}_{1}=1, \widetilde{N}_{2}^{*}=0$ or $\widetilde{N}_{1}=0$, $\widetilde{N}_{2}^{*}=1$, together with the corresponding 4 fermionic states $q=\left(\frac{1}{2},-\frac{1}{2}, \underline{\frac{1}{2}},-\frac{1}{2}\right)$ and the charge conjugate states correspond to two 6D hypermultiplets,

$$
C_{1}, \quad C_{2}
$$

\footnotetext{
${ }^{1}$ Underline denotes all permutations.
} 
They contain the two 'radion' fields of the small $\mathrm{G}_{2}$ and $\mathrm{SU}(3)$ tori as well as offdiagonal components of the metric and the tensor fields and the associated superpartners. The complex structure of the small dimensions is fixed. All 24 bosonic fields originate from the 64 bosonic states $\hat{G}_{M N}, \hat{B}_{M N}$ and $\hat{\Phi}$ in 10 dimensions. The remaining 40 bosonic states and their fermionic superpartners are projected out by the $\mathbb{Z}_{3}$ twist.

The massless physical states of the gauge sector,

$$
|q, p\rangle \equiv|q\rangle \otimes|p\rangle,
$$

have vanishing oscillator numbers and satisfy the projection conditions

$$
v_{3} \cdot q-V_{f} \cdot p=0 \bmod 1 .
$$

Here $V_{f}=2\left(V_{6}+n_{3} W_{3}\right)$ are the local $\mathbb{Z}_{3}$ gauge subtwists of the model. They differ by multiples of the $\mathbb{Z}_{3}$ Wilson line $W_{3}$ in the $\mathrm{SU}(3)$ plane, which distinguishes the three inequivalent fixed points labelled by $n_{3}=0,1,2$ (cf. Fig. 1). Eqs. (2.14) are equivalent to

$$
v_{3} \cdot q-V_{3} \cdot p=0 \bmod 1, \quad W_{3} \cdot p=0 \bmod 1,
$$

where the second condition reflects the fact that the finite extension of the $\mathrm{SU}(3)$ plane is neglected in the $6 \mathrm{D}$ effective field theory.

At each fixed point in the $\mathrm{SU}(3)$ plane the group $\mathrm{E}_{8} \times \mathrm{E}_{8}$ is broken to the subgroup $\mathrm{SO}(14) \times \mathrm{U}(1) \times[\mathrm{SO}(14) \times \mathrm{U}(1)]$, which is differently embedded into $\mathrm{E}_{8} \times \mathrm{E}_{8}$ at the different fixed points [18]. The brackets denote the subgroup of the second $E_{8}$. The $\mathrm{U}(1)$ factors are sometimes omitted; they can always be reconstructed since the rank of the gauge group is preserved. One easily verifies that the intersection of the three $\mathrm{E}_{8} \times \mathrm{E}_{8}$ subgroups, which yields the unbroken gauge group of the $6 \mathrm{D}$ theory, is given by

$$
\mathrm{G}_{6}=\mathrm{SU}(6) \times \mathrm{U}(1)^{3} \times\left[\mathrm{SU}(3) \times \mathrm{SO}(8) \times \mathrm{U}(1)^{2}\right],
$$

with the massless $\mathcal{N}=2$ vector multiplets

$$
(\mathbf{3 5} ; 1,1)+(1 ; \mathbf{8}, 1)+(1 ; 1,28)+5 \times(1 ; 1,1) .
$$

The massless vector states are obtained from the conditions (2.14) for $v_{3} \cdot q=0$. There are two further possibilities, $v_{3} \cdot q= \pm 1 / 3$ and $v_{3} \cdot q= \pm 2 / 3$, which lead to $\mathcal{N}=2$ hypermultiplets. A straightforward calculation yields the gauge multiplets

$$
(\mathbf{2 0} ; 1,1)+(1 ; 1, \mathbf{8})+\left(1 ; 1, \mathbf{8}_{s}\right)+\left(1 ; 1, \mathbf{8}_{c}\right)+4 \times(1 ; 1,1),
$$

with the U(1) charges listed in Table A.2.

In addition to the vector and hypermultiplets from the untwisted sector of the string, there are $6 \mathrm{D}$ bulk fields which originate from the twisted sectors $T_{2}$ and $T_{4}$ of 


\begin{tabular}{|c|c|c|c|}
\hline Sector & Multiplet & Representation & $\#$ \\
\hline \hline Gravity & Graviton & $G_{M N}$ & 1 \\
& Dilaton & $\Phi$ & 1 \\
& Hyper & $C_{1}, C_{2}$ & 2 \\
\hline Untwisted & Vector & $(\mathbf{3 5} ; 1,1)$ & 35 \\
& & $(1 ; \mathbf{8}, 1)$ & 8 \\
& & $(1 ; 1, \mathbf{2 8})$ & 28 \\
& & $5 \times(1 ; 1,1)$ & 5 \\
\hline Untwisted & Hyper & $(\mathbf{2 0} ; 1,1)$ & 20 \\
& & $(1 ; 1, \mathbf{8})+\left(1 ; 1, \mathbf{8}_{s}\right)+\left(1 ; 1, \mathbf{8}_{c}\right)$ & 24 \\
& & $4 \times(1 ; 1,1)$ & 4 \\
\hline Twisted & Hyper & $9 \times(\mathbf{6} ; 1,1)+9 \times(\overline{\mathbf{6}} ; 1,1)$ & 108 \\
& & $3 \times(1 ; 1, \mathbf{8})+3 \times(1 ; 1)+9 \times(1 ; \overline{\mathbf{3}} ; 1,1)$ & 54 \\
& & $36 \times(1 ; 1,1)+3 \times\left(1 ; 1, \mathbf{8}_{c}\right)$ & 72 \\
& & & 36 \\
\hline
\end{tabular}

Table 2.1: $\mathcal{N}=2$ supermultiplets of the $6 D$ theory: graviton, dilaton, 76 vector and 320 hypermultiplets. The non-Abelian symmetry group is $S U(6) \times[S U(3) \times S O(8)]$.

the $\mathbb{Z}_{6-\text { II }}$ model, corresponding to the twisted sectors $\hat{T}_{1}$ and $\hat{T}_{2}$ of the $\mathbb{Z}_{3}$ subtwist. The projection conditions for physical states are

$$
v_{3} \cdot\left(\widetilde{N}_{f}-\widetilde{N}_{f}^{*}\right)-v_{3} \cdot\left(q+v_{3}\right)+V_{f} \cdot\left(p+V_{f}\right)=0 \bmod 1,
$$

where $\widetilde{N}_{f}, \widetilde{N}_{f}^{*}$ are the integer oscillator numbers for left-moving modes localized at the fixed point $f$ (cf. [18]).

At each fixed point one has states with $\widetilde{N}_{f}=\widetilde{N}_{f}^{*}=0$, which yield $\mathcal{N}=2$ hypermultiplets $(\mathbf{1 4}, 1)$ and $(1, \mathbf{1 4})$. With respect to the 6D gauge group these multiplets form the reducible representations

$$
\begin{aligned}
& (\mathbf{1 4}, 1)=(\mathbf{6} ; 1,1)+(\overline{\mathbf{6}} ; 1,1)+2 \times(1 ; 1,1), \\
& (1, \mathbf{1 4})=(1 ; \mathbf{3}, 1)+(1 ; \overline{\mathbf{3}}, 1)+(1 ; 1, \hat{\mathbf{8}}) .
\end{aligned}
$$

At the three $\mathrm{SU}(3)$ fixed points, $(1 ; 1, \hat{\mathbf{8}})$ corresponds to $(1 ; 1, \mathbf{8}),\left(1 ; 1, \boldsymbol{8}_{s}\right)$ and $\left(1 ; 1, \mathbf{8}_{c}\right)$, respectively. Furthermore, there are oscillator states for the two small compact planes,

$$
\left|q+v_{3}\right\rangle \otimes \widetilde{\alpha}_{f-1}^{i}\left|p+V_{f}\right\rangle, \quad\left|q+v_{3}\right\rangle \otimes \widetilde{\alpha}_{f-1}^{* i}\left|p+V_{f}\right\rangle, \quad i=3,4,
$$

which yield two non-Abelian singlet hypermultiplets for each fixed point.

In addition to the three inequivalent fixed points in the $\mathrm{SU}(3)$ plane, there are three equivalent fixed points of the $\mathbb{Z}_{3}$ twist in the $\mathrm{G}_{2}$ plane. This yields a multiplicity of three for all hypermultiplets from the $T_{2}$ and $T_{4}$ sectors. All the multiplets of the 
6D theory are summarized in Table 2.1. The full listing including the $\mathrm{U}(1)$ charges is given in Appendix A.2.

Let us finally consider the interaction between vector and hypermultiplets. It is convenient to decompose all $\mathcal{N}=26 \mathrm{D}$ multiplets in terms of $\mathcal{N}=14 \mathrm{D}$ multiplets. The $6 \mathrm{D}$ vector multiplet splits into a pair of $4 \mathrm{D}$ vector and chiral multiplets, $A=(V, \phi)$, and a hypermultiplet consists of a pair of chiral multiplets, $H=\left(H_{L}, H_{R}\right)$; here $\phi$ and $H_{L}$ are left-handed, $H_{R}$ is right-handed. In flat space, the interaction lagrangian takes the simple form [25]

$$
\begin{aligned}
\mathcal{L}_{H}= & \int d^{4} \theta\left(H_{L}^{\dagger} e^{2 g V} H_{L}+H_{R}^{c \dagger} e^{-2 g V} H_{R}^{c}\right) \\
& +\int d^{2} \theta H_{R}^{c}(\partial+\sqrt{2} g \phi) H_{L}+\text { h.c. }
\end{aligned}
$$

After compactification to four dimensions, the first term yields the familiar gauge interactions, whereas the second term can give rise to Yukawa couplings. For the hypermultiplet $(20 ; 1,1)$ one obtains

$$
\mathcal{L}_{H} \supset \sqrt{2} g \int d^{2} \theta H_{R}^{c}(\mathbf{2 0}) \phi(\mathbf{3 5}) H_{L}(\mathbf{2 0})+\text { h.c. }
$$

The SU(6) 20-plet contains SU(5) 10- and $\overline{\mathbf{1 0}}$-plets, and the 35 -plet contains $\mathrm{SU}(5)$ 5 - and $\overline{5}$-plets. As we shall see in Section 6 , after projection onto 4D zero modes, Eq. (2.22) yields precisely the top Yukawa coupling. The Yukawa terms for the hypermultiplets $(\mathbf{6} ; 1,1)$ and $(\overline{\mathbf{6}} ; 1,1)$,

$$
\mathcal{L}_{H} \supset \sqrt{2} g \int d^{2} \theta\left(H_{R}^{c}(\mathbf{6}) \phi(\mathbf{3 5}) H_{L}(\mathbf{6})+H_{R}^{c}(\overline{\mathbf{6}}) \phi(\mathbf{3 5}) H_{L}(\overline{\mathbf{6}})\right)+\text { h.c. }
$$

will be important for the decoupling of exotic states in Section 5 .

\section{3. $\mathbb{Z}_{2}$ Compactification to Four Dimensions}

The compactification from six to four dimensions on a $\mathbb{Z}_{2}$ orbifold leads to four additional fixed points in the $\mathrm{SO}(4)$ plane and to further projection conditions for physical massless states. The fixed points are labelled by $\left(n_{2}, n_{2}^{\prime}\right)=(0,0),(0,1),,(1,0),(1,1)$ (cf. Fig. 1). Due to the Wilson line $W_{2}$, they come in two pairs of equivalent fixed points, and the projection conditions only depend on $n_{2}$ and not on $n_{2}^{\prime}$.

At the fixed points, half of the supersymmetry generators are broken and only $\mathcal{N}=1$ supersymmetry remains unbroken. For the gravitational and gauge multiplets of the untwisted sector the projection conditions are [18]

$$
v_{2} \cdot\left(\tilde{N}-\tilde{N}^{*}\right)-v_{2} \cdot q+V_{f} \cdot p=0 \bmod 1,
$$

where $v_{2}=3 v_{6}$, and $V_{f}=3 V_{6}+n_{2} W_{2}$ are the local twists at the fixed points $n_{2}=0,1$ in the $\mathrm{SO}(4)$ plane. 


\begin{tabular}{|c|c|}
\hline$n_{2}$ & Gauge group \\
\hline \hline 0 & $\mathrm{SU}(5) \times \mathrm{U}(1)^{4} \times\left[\mathrm{SU}(3) \times \mathrm{SO}(8) \times \mathrm{U}(1)^{2}\right]$ \\
\hline 1 & $\mathrm{SU}(2) \times \mathrm{SU}(4) \times \mathrm{U}(1)^{4} \times\left[\mathrm{SU}(2)^{\prime} \times \mathrm{SU}(4)^{\prime} \times \mathrm{U}(1)^{4}\right]$ \\
\hline \hline$\cap$ & $\mathrm{SU}(3) \times \mathrm{SU}(2) \times \mathrm{U}(1)^{5} \times\left[\mathrm{SU}(2)^{\prime} \times \mathrm{SU}(4)^{\prime} \times \mathrm{U}(1)^{4}\right]$ \\
\hline
\end{tabular}

Table 3.1: List of the local gauge groups and their intersection.

In this paper we consider an anisotropic orbifold where the $\mathrm{SO}(4)$ plane is much larger than the $\mathrm{G}_{2}$ and $\mathrm{SU}(3)$ planes. The Kaluza-Klein states of the $\mathrm{SO}(4)$ plane can be included in an effective field theory below the string scale by considering fields in the two large compact dimensions instead of 4D zero modes which are assumed to be constant in the compact dimensions. For the $\mathbb{Z}_{2}$ twist, one has (cf. [18]) $\left(\theta^{3}, l_{f}\right)\left(z_{f}^{3}+z^{3}\right)=z_{f}^{3}-z^{3}$, where $\left(\theta^{3}, l_{f}\right)$ is the space group element of the fixed point $f$ and $z^{3}=y^{5}+\mathrm{i} y^{6}$ is the complex coordinate in the $\mathrm{SO}(4)$ plane. The projection conditions (3.1) for the massless states then become local projection conditions for fields in the compact dimensions,

$$
\begin{aligned}
P_{f}: \phi\left(y_{f}+y\right) & =\eta_{f}(\phi) \phi\left(y_{f}-y\right) \\
\eta_{f}(\phi) & =\exp \left\{2 \pi \mathrm{i}\left(v_{2} \cdot\left(\tilde{N}-\tilde{N}^{*}-q\right)+V_{f} \cdot p\right)\right\} .
\end{aligned}
$$

The momenta $p, q$ and the oscillator number $\tilde{N}-\tilde{N}^{*}$ of the states determine the quantum numbers of the corresponding fields $\phi$, and $\eta_{f}(\phi)= \pm 1$. Only fields which have positive parity at all fixed points have zero modes.

As an example, consider the 6D metric

$$
\mathrm{d} s^{2}=g_{M N} \mathrm{~d} x^{M} \mathrm{~d} x^{N}=g_{\mu \nu} \mathrm{d} x^{\mu} \mathrm{d} x^{\nu}+2 g_{\mu m} \mathrm{~d} x^{\mu} \mathrm{d} y^{m}+g_{m n} \mathrm{~d} y^{m} \mathrm{~d} y^{n},
$$

where $x^{\mu}$ and $y^{m}$ are the coordinates of 4D Minkowski space and the two compact dimensions, respectively. One easily obtains from Eqs. (2.8) and (3.2) the projection conditions

$$
g_{\mu \nu}(x, y)=g_{\mu \nu}(x,-y), \quad g_{\mu m}(x, y)=-g_{\mu m}(x,-y), \quad g_{m n}(x, y)=g_{m n}(x,-y) .
$$

The $4 \mathrm{D}$ zero mode $g_{\mu \nu}(x)$ is part of the $\mathcal{N}=1$ supergravity multiplet $\left(g_{\mu \nu}, \psi_{\mu}\right)$ while the three degrees of freedom in $g_{m n}(x)$ join with $B_{56}$ to form the moduli multiplets $T$ and $S$ containing the radion field and the complex structure of the torus.

The projection conditions for the $\mathcal{N}=2$ vector multiplets $A$ are most conveniently expressed in terms of the corresponding $\mathcal{N}=1$ vector $(V)$ and chiral $(\phi)$ multiplets, $A=(V, \phi)$, which are elements of the Lie algebra of the 6D bulk gauge group. The unbroken gauge group at the fixed point $f$ is determined by the condition

$$
p \cdot V_{f}=0 \bmod 1 \text {. }
$$


At the fixed points $n_{2}=0$ and $n_{2}=1$ in the $\mathrm{SO}(4)$ plane, the bulk gauge group $\mathrm{SU}(6) \times[\mathrm{SU}(3) \times \mathrm{SO}(8)]$ is broken to subgroups containing $\mathrm{SU}(5) \times\left[\mathrm{SU}(2)^{\prime} \times \mathrm{SU}(4)^{\prime}\right]$ and $\mathrm{SU}(2) \times \mathrm{SU}(4) \times\left[\mathrm{SU}(2)^{\prime} \times \mathrm{SU}(4)^{\prime}\right]$, respectively. At the two fixed points the conditions for the vector and chiral multiplets are given by

$$
P_{f} V\left(x, y_{f}-y\right) P_{f}=V\left(x, y_{f}+y\right), \quad P_{f} \phi\left(x, y_{f}-y\right) P_{f}=-\phi\left(x, y_{f}+y\right),
$$

where $P_{f}$ is the $\mathbb{Z}_{2}$ parity matrix. Again only $\mathcal{N}=1$ supersymmetry remains unbroken. As an example, for the $\mathrm{SU}(6)$ factor, one has $P_{0}=\operatorname{diag}(1,1,1,1,1,-1)$ at $n_{2}=0$, and $P_{1}=\operatorname{diag}(1,1,-1,-1,-1,-1)$ at $n_{2}=1$. The decomposition of the bulk gauge fields with respect to the locally unbroken subgroups, together with all $\mathrm{U}(1)$ charges, are listed in Tables 3.2 and 3.3. For the unbroken subgroup, vectors have positive and scalars negative parity; for the broken generators the situation is reversed.

At the fixed point $n_{2}=0$ the GUT group $\mathrm{SU}(5) \times \mathrm{U}(1)$ is unbroken, and the $\mathcal{N}=2$ vector multiplet 35 of $\mathrm{SU}(6)$ splits into the $\mathcal{N}=1$ vector multiplets $\mathbf{2 4}+\mathbf{1}$ with positive parity and the $\mathcal{N}=1$ chiral multiplets $\mathbf{5}+\overline{\mathbf{5}}$ with positive parity from the coset $\mathrm{SU}(6) /(\mathrm{SU}(5) \times \mathrm{U}(1))$. From Table 3.3 one reads off that the projection condition at the fixed point $n_{2}=1$ projects out the color triplets from both the 5and the $\overline{\mathbf{5}}$-plets. This is the well known doublet-triplet splitting of orbifold GUTs. As we shall discuss in Section 5, the remaining SU(2) doublets can play the role of Higgs or lepton doublets in the 4D effective theory.

The $\mathcal{N}=2$ hypermultiplets $H$ consist of pairs of $\mathcal{N}=1$ left- and right-chiral multiplets, $H=\left(H_{L}, H_{R}\right)$. For the projection conditions one finds

$$
P_{f} H_{L}\left(x, y_{f}-y\right)=\eta_{f} H_{L}\left(x, y_{f}+y\right), \quad P_{f} H_{R}\left(x, y_{f}-y\right)=-\eta_{f} H_{R}\left(x, y_{f}+y\right)
$$

where $P_{f}$ is now a matrix in the representation of $H$, and $\eta_{f}$ has to be calculated using Eq. (3.2). The parities for the hypermultiplets from the untwisted sector, decomposed with respect to the unbroken groups at the fixed points $n_{2}=0$ and $n_{2}=1$ are listed in the Tables 3.4 and 3.5.

Zero modes with standard model quantum numbers are contained in two $\mathcal{N}=1$ chiral multiplets which are $\mathrm{SU}(5)$ 10-plets,

$$
H_{L}=(\mathbf{1 0} ; 1,1), \quad H_{R}^{c}=\left(\overline{\mathbf{1 0}}^{c} ; 1,1\right) .
$$

From the Tables 3.4 and 3.5 one easily verifies that the projection conditions at the fixed point $n_{2}=1$ yield the following quark-lepton states as $4 \mathrm{D}$ zero modes:

$$
10:(3,2)=q ; \quad \overline{\mathbf{1 0}}^{c}:(\overline{3}, 1)=u^{c},(1,1)=e^{c} .
$$

Together, the zero modes have again the quantum numbers of one SU(5) 10-plet. However, as we shall see in Section 6, it is crucial for their Yukawa couplings that they originate from two distinct $\mathrm{SU}(5)$ 10-plets. 


\begin{tabular}{|c||c||c|c||c|}
\hline Bulk & $n_{2}=0$ & $\mathrm{~V}$ & $\phi$ & $t_{6}^{0}$ \\
\hline \hline$(\mathbf{3 5} ; 1,1)$ & $(\mathbf{2 4} ; 1,1)$ & + & - & 0 \\
& $(\mathbf{5} ; 1,1)$ & - & + & -6 \\
& $(\overline{\mathbf{5}} 1,1)$ & - & + & 6 \\
& $(1 ; 1,1)$ & + & - & 0 \\
\hline$(1 ; \mathbf{8}, 1)$ & $(1 ; \mathbf{8}, 1)$ & + & - & 0 \\
\hline$(1 ; 1, \mathbf{2 8})$ & $(1 ; 1, \mathbf{2 8})$ & + & - & 0 \\
\hline
\end{tabular}

Table 3.2: Local decomposition of vector multiplets at $n_{2}=0$.

\begin{tabular}{|c||c||c|c||c|c|c|}
\hline Bulk & $n_{2}=1$ & $\mathrm{~V}$ & $\phi$ & $t_{6}^{1}$ & $t_{7}$ & $t_{8}$ \\
\hline \hline$(\mathbf{3 5} ; 1,1)$ & $(\mathbf{3}, 1 ; 1,1)$ & + & - & 0 & 0 & 0 \\
& $(1, \mathbf{1 5} ; 1,1)$ & + & - & 0 & 0 & 0 \\
& $(\mathbf{2}, \mathbf{4} ; 1,1)$ & - & + & 15 & 0 & 0 \\
& $(\mathbf{2}, \overline{\mathbf{4}} ; 1,1)$ & - & + & -15 & 0 & 0 \\
& $(1,1 ; 1,1)$ & + & - & 0 & 0 & 0 \\
\hline$(1 ; \mathbf{8}, 1)$ & $(1,1 ; \mathbf{3}, 1)$ & + & - & 0 & 0 & 0 \\
& $(1,1 ; \mathbf{2}, 1)$ & - & + & 0 & 3 & 0 \\
& $(1,1 ; \mathbf{2}, 1)$ & - & + & 0 & -3 & 0 \\
& $(1,1 ; 1,1)$ & + & - & 0 & 0 & 0 \\
\hline$(1 ; 1, \mathbf{2 8})$ & $(1,1 ; 1, \mathbf{1 5})$ & + & - & 0 & 0 & 0 \\
& $(1,1 ; 1, \mathbf{6})$ & - & + & 0 & 0 & 2 \\
& $(1,1 ; 1, \mathbf{6})$ & - & + & 0 & 0 & -2 \\
& $(1,1 ; 1,1)$ & + & - & 0 & 0 & 0 \\
\hline
\end{tabular}

Table 3.3: Local decomposition of vector multiplets at $n_{2}=1$.

As discussed in the previous section, the $\mathcal{N}=2$ hypermultiplets from the $T_{2} / T_{4}$ sector are bulk fields in the $\mathrm{SO}(4)$ plane, but localized in the $\mathrm{G}_{2}$ and $\mathrm{SU}(3)$ planes. With respect to the bulk gauge group they transform as $(\mathbf{6} ; 1,1),(\overline{\mathbf{6}} ; 1,1),(1 ; \mathbf{3}, 1)$, $(1 ; \overline{\mathbf{3}}, 1),(1 ; 1, \mathbf{8}),\left(1 ; 1, \boldsymbol{8}_{c}\right),\left(1 ; 1, \boldsymbol{8}_{s}\right)$ and $(1 ; 1,1)$. One can form linear combinations of the states localized at the equivalent fixed points in the $\mathrm{G}_{2}$ plane, which are eigenstates of the $\mathbb{Z}_{2}$ twist, $\Theta^{3}\left|q_{\gamma}\right\rangle=\exp \left(2 \pi \mathrm{i} q_{\gamma}\right)\left|q_{\gamma}\right\rangle$. For the twisted sector fields the projection conditions depend on the phase $q_{\gamma}$, and the parities $\eta_{f}(\phi)$ are given by

$$
\eta_{f}(\phi)=\exp \left\{2 \pi \mathrm{i}\left(v_{2} \cdot\left(\tilde{N}-\tilde{N}^{*}-q\right)+V_{f} \cdot p+q_{\gamma}\right)\right\} .
$$

For the $T_{2} / T_{4}$ twisted states $q_{\gamma}$ takes the values $0,1 / 2,1$. The corresponding 6 parities for all hypermultiplets $H=\left(H_{L}, H_{R}\right)$ at the fixed points $n_{2}=0$ and $n_{2}=1$ are listed in Tables A.4 A.7. 


\begin{tabular}{|c||c||c|c||c|c|c|c|c|c||c|}
\hline Bulk & $n_{2}=0$ & $H_{L}$ & $H_{R}$ & $t_{6}^{0}$ & $t_{1}$ & $t_{2}$ & $t_{3}$ & $t_{4}$ & $t_{5}$ & \\
\hline \hline$(\mathbf{2 0} ; 1,1)$ & $(\mathbf{1 0} ; 1,1)$ & + & - & 3 & $-\frac{1}{2}$ & $\frac{1}{2}$ & 0 & 0 & 0 & \\
& $(\overline{\mathbf{1 0}} ; 1,1)$ & - & + & -3 & $-\frac{1}{2}$ & $\frac{1}{2}$ & 0 & 0 & 0 & \\
\hline$(1 ; 1, \mathbf{8})$ & $(1 ; 1, \mathbf{8})$ & - & + & 0 & 0 & 0 & 0 & -1 & 0 & \\
\hline$\left(1 ; 1, \mathbf{8}_{\mathbf{s}}\right)$ & $\left(1 ; 1, \mathbf{8}_{\mathbf{s}}\right)$ & + & - & 0 & 0 & 0 & 0 & $\frac{1}{2}$ & $\frac{3}{2}$ & \\
\hline$\left(1 ; 1, \mathbf{8}_{\mathbf{c}}\right)$ & $\left(1 ; 1, \mathbf{8}_{\mathbf{c}}\right)$ & + & - & 0 & 0 & 0 & 0 & $\frac{1}{2}$ & $-\frac{3}{2}$ & \\
\hline$(1 ; 1,1)$ & $(1 ; 1,1)$ & - & + & 0 & $\frac{1}{2}$ & $\frac{1}{2}$ & 3 & 0 & 0 & $U_{1}$ \\
\hline$(1 ; 1,1)$ & $(1 ; 1,1)$ & + & - & 0 & $\frac{1}{2}$ & $\frac{1}{2}$ & -3 & 0 & 0 & $U_{2}$ \\
\hline$(1 ; 1,1)$ & $(1 ; 1,1)$ & + & - & 0 & 1 & -1 & 0 & 0 & 0 & $U_{3}$ \\
\hline$(1 ; 1,1)$ & $(1 ; 1,1)$ & + & - & 0 & -1 & -1 & 0 & 0 & 0 & $U_{4}$ \\
\hline
\end{tabular}

Table 3.4: Local decomposition of untwisted hypermultiplets at $n_{2}=0$.

\begin{tabular}{|c||c||c|c||c|c|c|c|c|c|c|c||c|}
\hline Bulk & $n_{2}=1$ & $H_{L}$ & $H_{R}$ & $t_{6}^{1}$ & $t_{7}$ & $t_{8}$ & $t_{1}$ & $t_{2}$ & $t_{3}$ & $t_{4}$ & $t_{5}$ & \\
\hline \hline$(\mathbf{2 0} ; 1,1)$ & $(\mathbf{2}, \mathbf{6} ; 1,1)$ & - & + & 0 & 0 & 0 & $-\frac{1}{2}$ & $\frac{1}{2}$ & 0 & 0 & 0 & \\
& $(1, \mathbf{4} ; 1,1)$ & + & - & -15 & 0 & 0 & $-\frac{1}{2}$ & $\frac{1}{2}$ & 0 & 0 & 0 & \\
& $(1, \overline{\mathbf{4}} ; 1,1)$ & + & - & 15 & 0 & 0 & $-\frac{1}{2}$ & $\frac{1}{2}$ & 0 & 0 & 0 & \\
\hline$(1 ; 1, \mathbf{8})$ & $(1,1 ; 1, \mathbf{4})$ & - & + & 0 & 0 & -1 & 0 & 0 & 0 & -1 & 0 & \\
& $(1,1 ; 1, \overline{\mathbf{4}})$ & + & - & 0 & 0 & 1 & 0 & 0 & 0 & -1 & 0 & \\
\hline$\left(1 ; 1, \mathbf{8}_{\mathbf{c}}\right)$ & $(1,1 ; 1, \mathbf{6})$ & - & + & 0 & 0 & 0 & 0 & 0 & 0 & $\frac{1}{2}$ & $-\frac{3}{2}$ & \\
& $(1,1 ; 1,1)$ & + & - & 0 & 0 & 2 & 0 & 0 & 0 & $\frac{1}{2}$ & $-\frac{3}{2}$ & \\
& $(1,1 ; 1,1)$ & + & - & 0 & 0 & -2 & 0 & 0 & 0 & $\frac{1}{2}$ & $-\frac{3}{2}$ & \\
\hline$\left(1 ; 1, \mathbf{8}_{\mathbf{s}}\right)$ & $(1,1 ; 1, \mathbf{4})$ & - & + & 0 & 0 & 1 & 0 & 0 & 0 & $\frac{1}{2}$ & $\frac{3}{2}$ & \\
& $(1,1 ; 1, \overline{\mathbf{4}})$ & + & - & 0 & 0 & -1 & 0 & 0 & 0 & $\frac{1}{2}$ & $\frac{3}{2}$ & \\
\hline$(1 ; 1,1)$ & $(1,1 ; 1,1)$ & - & + & 0 & 0 & 0 & $\frac{1}{2}$ & $\frac{1}{2}$ & 3 & 0 & 0 & $U_{1}$ \\
\hline$(1 ; 1,1)$ & $(1,1 ; 1,1)$ & - & + & 0 & 0 & 0 & $\frac{1}{2}$ & $\frac{1}{2}$ & -3 & 0 & 0 & $U_{2}$ \\
\hline$(1 ; 1,1)$ & $(1,1 ; 1,1)$ & - & + & 0 & 0 & 0 & 1 & -1 & 0 & 0 & 0 & $U_{3}$ \\
\hline$(1 ; 1,1)$ & $(1,1 ; 1,1)$ & - & + & 0 & 0 & 0 & -1 & -1 & 0 & 0 & 0 & $U_{4}$ \\
\hline
\end{tabular}

Table 3.5: Local decomposition of untwisted hypermultiplets at $n_{2}=1$.

The 6D theory contains 9 hypermultiplets of SU(5) 5 -plets and 9 hypermultiplets of $\overline{\mathbf{5}}$-plets. Each hypermultiplet contains a pair of $\mathbf{5}$ and $\overline{\mathbf{5}} \mathcal{N}=1$ chiral multiplets. 
As Table A.4 shows, the positive parities select from each triplet of hypermultiplets, with $q_{\gamma}=0,1 / 2,1$, a chiral combination of 5-plets: one 5 and two $\overline{5}$ 's or two $\mathbf{5}$ 's and one $\overline{\mathbf{5}}$. The projection conditions at $n_{2}=1$ then leave as $4 \mathrm{D}$ zero modes from each 5- or $\overline{\mathbf{5}}$-plet either the $\mathrm{SU}(3)$ triplet or the $\mathrm{SU}(2)$ doublet. In this way a spectrum of massless states is generated which is chiral with respect to the standard model group.

The $\mathbb{Z}_{2}$ orbifolding leads from the $\mathbb{Z}_{3}$ orbifold model of Section 2 to a $\mathbb{Z}_{6}$ orbifold model, and therefore to new twisted sectors $T_{1} / T_{5}$ and $T_{3}$. The massless states are obtained from the corresponding mass equations (cf. [18]) with $k=1$ and $k=3$, respectively. In the $T_{3}$ sector one can choose a basis of eigenstates of the $\mathbb{Z}_{3}$ twist, $\Theta^{2}\left|q_{\gamma}\right\rangle=\exp \left(2 \pi \mathrm{i} q_{\gamma}\right)\left|q_{\gamma}\right\rangle$, with $q_{\gamma}=0,1 / 3,-1 / 3,1$ (cf. [18]). The projection conditions for physical states now involve the parities

$$
\eta_{f}(\phi)=\exp \left\{2 \pi \mathrm{i}\left(v_{3} \cdot\left(\tilde{N}-\tilde{N}^{*}-q\right)+V_{f} \cdot p+q_{\gamma}\right)\right\} .
$$

The states are bulk fields in the $\mathrm{SU}(3)$ plane, whose extension we neglect, but localized in the $\mathrm{G}_{2}$ and $\mathrm{SO}(4)$ planes. All massless states from the $T_{1} / T_{5}$ and $T_{3}$ sectors at the fixed points $n_{2}=0$ and $n_{2}=1$ are listed in Tables A.8 and A.9.

At both fixed points with $n_{2}=0$, one standard model family with SU(5) quantum numbers $\overline{\mathbf{5}}+\mathbf{1 0}$ occurs. All other states are standard model singlets. On the contrary, there are no standard model singlets at the fixed point $n_{2}=1$, but only color singlets with exotic $\mathrm{SU}(2) \times \mathrm{U}(1)$ quantum numbers.

So far we have ignored the localization number $n_{2}^{\prime}=0,1$ of the fixed points in the $\mathrm{SO}(4)$ plane, since it just leads to a doubling of the states localized at $n_{2}=0,1$. Altogether, we have a rather simple picture for the standard model non-singlet states: There are two quark-lepton families localized at

$$
n_{2}=0, n_{2}^{\prime}=0,1: \quad \overline{\mathbf{5}}+\mathbf{1 0} .
$$

From the bulk fields, vector and hypermultiplets, we have

$$
11 \times \overline{\mathbf{5}}+9 \times \mathbf{5}+\mathbf{1 0}+\overline{\mathbf{1 0}}^{c} .
$$

The spectrum is chiral and looks like four quark-lepton families plus 9 pairs of 5's and $\overline{\mathbf{5}}$ 's. However, the projection conditions at the $n_{2}=1$ fixed points eliminate half of the bulk fields, so that one is left with three quark-lepton families and several vector-like pairs of $\mathrm{SU}(3)$ triplets and $\mathrm{SU}(2)$ doublets which can accommodate a pair of Higgs doublets. Which $\overline{5}$ 's contain the quark and lepton states of the third family, and which one the Higgs doublet depends on the chosen vacuum. At the fixed points $n_{2}=1$ there are additional localized states with exotic quantum numbers. Using the Tables 3.2-3.5 and A.4-A.9, one can check that the spectrum of zero modes obtained in [18] is reproduced.

The determination of possible supersymmetric vacua, where some of the standard model singlet fields acquire large VEVs, is discussed in Sections 5 and 6 . In such 


\begin{tabular}{|c|l|c|c|c|}
\hline $\mathrm{U}(1)$ & Generator Embedding into $E_{8} \times E_{8}$ & Bulk & $n_{2}=0$ & $n_{2}=1$ \\
\hline \hline$t_{1}$ & $(0,1,0,0,0,0,0,0)(0,0,0,0,0,0,0,0)$ & $\sqrt{ }$ & $\sqrt{ }$ & $\sqrt{ }$ \\
\hline$t_{2}$ & $(0,0,1,0,0,0,0,0)(0,0,0,0,0,0,0,0)$ & $\sqrt{ }$ & $\sqrt{ }$ & $\sqrt{ }$ \\
\hline$t_{3}$ & $(1,0,0,1,1,1,1,1)(0,0,0,0,0,0,0,0)$ & $\sqrt{ }$ & $\sqrt{ }$ & $\sqrt{ }$ \\
\hline$t_{4}$ & $(0,0,0,0,0,0,0,0)(1,0,0,0,0,0,0,0)$ & $\sqrt{ }$ & $\sqrt{ }$ & $\sqrt{ }$ \\
\hline$t_{5}$ & $(0,0,0,0,0,0,0,0)(0,1,1,1,0,0,0,0)$ & $\sqrt{ }$ & $\sqrt{ }$ & $\sqrt{ }$ \\
\hline$t_{6}^{0}$ & $(5,0,0,-1,-1,-1,-1,-1)(0,0,0,0,0,0,0,0)$ & $\times$ & $\sqrt{ }$ & $\times$ \\
\hline$t_{6}^{1}$ & $(5,0,0,-10,-10,5,5,5)(0,0,0,0,0,0,0,0)$ & $\times$ & $\times$ & $\sqrt{ }$ \\
\hline$t_{7}$ & $(0,0,0,0,0,0,0,0)(0,1,1,-2,0,0,0,0)$ & $\times$ & $\times$ & $\sqrt{ }$ \\
\hline$t_{8}$ & $(0,0,0,0,0,0,0,0)(0,0,0,0,-1,-1,-1,1)$ & $\times$ & $\times$ & $\sqrt{ }$ \\
\hline$t_{\mathrm{an}}^{0}$ & $(5,0,-4,-1,-1,-1,-1,-1)(5,-1,-1,-1,0,0,0,0)$ & & $\sqrt{ }$ & \\
\hline$t_{\mathrm{an}}^{1}$ & $(1,3,-1,1,1,1,1,1)(-4,4,4,4,0,0,0,0)$ & & & $\sqrt{ }$ \\
\hline$t_{\mathrm{an}}^{(4 \mathrm{~d})}$ & $\left(\frac{11}{6}, \frac{1}{2},-\frac{3}{2},-\frac{1}{6},-\frac{1}{6},-\frac{1}{6},-\frac{1}{6},-\frac{1}{6}\right)\left(1, \frac{1}{3}, \frac{1}{3}, \frac{1}{3}, 0,0,0,0\right)$ & & & \\
\hline
\end{tabular}

Table 4.1: Definition of the $U(1)$ generators. The last three columns indicate whether the generator is part of a non-Abelian group $(\times)$ or commutes with the semi-simple group $(\sqrt{ })$ in the bulk and at the fixed points. The anomalous $U(1)$ 's are linear combinations of the commuting $U(1)$ 's at the fixed point specified by the superscript or in four dimensions; they are denoted by $t_{\mathrm{an}}^{0}, t_{\mathrm{an}}^{1}$ and $t_{\mathrm{an}}^{(4 d)}$, respectively.

vacua, unwanted $\mathrm{SU}(3)$ triplets and $\mathrm{SU}(2)$ can be decoupled. The positive and negative parities at the fixed points $n_{2}=0,1$, listed in the Tables 3.2-3.5 and A.4A.7 are also needed to check the cancellation of anomalies for the constructed $6 \mathrm{D}$ supergravity theory.

\section{Anomalies}

Anomalies of field theories on orbifolds are well understood [26], and also the sixdimensional case has been discussed in detail [21,22,27-29]. In general the orbifold anomaly has bulk and brane contributions. While the bulk terms are already present in the torus compactification, the localized anomalies crucially depend on the projection conditions at the orbifold fixed points and the twisted sectors of the orbifold. Thus the requirement that all anomalies of the model can be cancelled imposes highly non-trivial conditions on the spectrum. In the present model their fulfillment is guaranteed by the fact that it has been derived from string theory, which automatically provides the right Green-Schwarz terms for anomaly cancellation [20]. In this section we apply its six-dimensional version $[21,22]$ to our effective $T^{2} / \mathbb{Z}_{2}$ orbifold model. 


\subsection{Anomalies and the Green-Schwarz Mechanism}

Gauge anomalies require chiral fermions ${ }^{2}$, so they can occur in any even dimension. Gravitational anomalies ${ }^{3}$, on the other hand, only arise in $4 k+2$ dimensions $(k=0,1, \ldots)$, hence they will appear in the bulk theory, but not on the branes.

The anomaly $\mathcal{A}$ is defined as the (nonvanishing) gauge variation of the effective action, $\mathcal{A}(\Lambda)=\delta_{\Lambda} \Gamma$. It can be computed from the anomaly polynomial, a (formal) closed and gauge invariant $(d+2)$-form $I_{d+2}$, via the Stora-Zumino descent equations $[30]$,

$$
\mathcal{A}(\Lambda) \propto \int I_{d}^{(1)}, \quad \mathrm{d} I_{d}^{(1)}=\delta_{\Lambda} I_{d+1}^{(0)}, \quad \mathrm{d} I_{d+1}^{(0)}=I_{d+2}
$$

where the superscript indicates the order in the parameter $\Lambda . I_{d+2}$ is a polynomial in traces of powers of the Riemann and Yang-Mills field strength tensors $R$ and $F_{I}$, interpreted as matrix-valued two-forms $\frac{1}{2} R_{\mu \nu a}{ }^{b} \mathrm{~d} x^{\mu} \mathrm{d} x^{\nu}$ and $\frac{1}{2} F_{I \mu \nu i}{ }^{j} \mathrm{~d} x^{\mu} \mathrm{d} x^{\nu}$. They are derived from spin and gauge connection one-forms as $R=\mathrm{d} \Omega+\Omega^{2}$ and $F_{I}=\mathrm{d} A_{I}+A_{I}^{2}$, where $I$ labels the factors of the gauge group. Here $a, b$ are indices in the vector representation of $S O(1, d-1), i, j$ are indices of some representation of the gauge group, and wedge products of forms are understood. Expressions of the form $\operatorname{tr} F_{I}^{n}$ or $\operatorname{tr} R^{n}$, the building blocks of $I_{d+2}$, are always closed and gauge invariant. Their coefficients in the anomaly polynomial depend on the numbers, representations and charges of the fermions under the respective gauge groups.

For the Green-Schwarz mechanism to cancel the anomalies, we exploit the transformation properties of the two-form $B_{2}=\frac{1}{2} B_{\mu \nu} \mathrm{d} x^{\mu} \mathrm{d} x^{\nu}$. Its variation under gauge and Lorentz transformations with parameters $\Lambda_{I}$ and $\Theta$ is

$$
\delta B_{2}=\operatorname{tr}(\Theta \mathrm{d} \Omega)-\sum_{I} \alpha_{I} \operatorname{tr}\left(\Lambda_{I} \mathrm{~d} A_{I}\right) .
$$

The coefficients $\alpha_{I}$ are $\alpha_{\mathrm{SU}(N)}=2$ and $\alpha_{\mathrm{SO}(N)}=1$ (the $U(1)$ coefficients are normalization dependent). The crucial feature of this transformation is that $\delta B_{2}$ itself is the descent of the closed and gauge invariant four-form

$$
X_{4}=\operatorname{tr} R^{2}-\sum_{I} \alpha_{I} \operatorname{tr} F_{I}^{2}
$$

such that the 3-form field strength $H_{3}=\mathrm{d} B_{2}-X_{3}^{(0)}$ associated with $B_{2}$ is invariant. By adding appropriate interaction terms of the $B$-field to the action it is now possible to achieve a complete cancellation of the reducible anomalies.

\footnotetext{
${ }^{2}$ Also (anti)self-dual tensor fields can contribute. Since in our model there is one tensor field of each type, their effects cancel.

${ }^{3}$ Anomalies in local Lorentz transformations and in general coordinate transformations are equivalent in the sense that the anomaly can be shifted from one to the other by local counterterms. We will consider anomalies in local Lorentz transformations and refer to those as gravitational.
} 
For $T^{2} / \mathbb{Z}_{2}$ orbifolds, the total anomaly polynomial $I_{8}$ is of the form

$$
I_{8}=\frac{1}{2} I_{8}^{\text {bulk }}+\sum_{f} I_{6}^{f} \delta^{2}\left(y-y_{f}\right) \mathrm{d} y^{5} \mathrm{~d} y^{6},
$$

where $I_{8}^{\text {bulk }}$ is the anomaly polynomial on $\mathbb{R}^{1,3} \times T^{2}$, and $I_{6}^{f}$ is the local anomaly polynomial at the fixed point $f . I_{6}^{f}$ receives two kinds of contributions: Branelocalized fields and bulk fields surviving the orbifold projection at this particular fixed point. The latter, however, contribute with a factor of $\frac{1}{4}$ because the orbifold contains four fixed points. The factor $\frac{1}{2}$ in (4.4) enters since the fundamental domain of the orbifold is half the one of the torus. These anomalies can be cancelled by the Green-Schwarz mechanism if $I_{8}$ is reducible, i.e., if it factorizes into a product involving $X_{4}$. For the components this means

$$
I_{8}^{\text {bulk }}=\beta X_{4} Y_{4}, \quad I_{6}^{f}=\alpha X_{4}^{f} Y_{2}^{f} .
$$

Here $X_{4}^{f}$ follows from $X_{4}$ by projection onto the local gauge group, and we have pulled out factors $\alpha=\frac{i}{48(2 \pi)^{3}}$ and $\beta=\frac{-i}{16(2 \pi)^{3}}$. Since $\operatorname{tr} R=\operatorname{tr} F=0$ for nonAbelian gauge groups, the localized two-forms $Y_{2}^{f}$ can only be linear combinations of $\mathrm{U}(1)$ field strengths, which can be redefined as $Y_{2}^{f}=c^{f} F^{f}=c^{f} \mathrm{~d} A^{f}$. $A^{f}$ and the corresponding generator are referred to as the anomalous $\mathrm{U}(1)$ at the fixed point $f$.

If the anomaly polynomial factorizes in the required way, the total anomaly $\mathcal{A}=\int I_{6}^{(1)}$ descends from (4.4) and is cancelled by variation of the Green-Schwarz action [22],

$$
\begin{aligned}
S_{\mathrm{GS}}=\int\{ & -\left(\frac{\beta}{2} Y_{3}^{(0)}+\alpha \sum_{f} c^{f} A^{f} \delta^{2}\left(y-y_{f}\right) \mathrm{d} y^{5} \mathrm{~d} y^{6}\right) \mathrm{d} B \\
+ & \left.\left(\frac{\beta}{4} Y_{3}^{(0)}+\frac{\alpha}{3} \sum_{f} c^{f} A^{f} \delta^{2}\left(y-y_{f}\right) \mathrm{d} y^{5} \mathrm{~d} y^{6}\right) X_{3}^{(0)}\right\} .
\end{aligned}
$$

\subsection{Bulk Anomalies}

We now check the cancellation of bulk anomalies in the model at hand. It is convenient to split the gauge group index as $I=(A, u)$, with $A, B, \ldots$ running over the non-Abelian factors, i.e. $\mathrm{SU}(6), \mathrm{SU}(3)$ and $\mathrm{SO}(8)$, while $u, v, \ldots=1, \ldots, 5$ label the U(1) factors. The anomaly polynomial for the six-dimensional case is given in Ref. [21]. Here we first check that the irreducible pieces cancel and then show that the remaining parts factorize as in (4.5).

There are three contributions in the anomaly polynomial which cannot be reducible:

- The most severe constraint arises from the quartic pure gravitational anomaly. The corresponding term in the anomaly polynomial is

$$
(244+y-s) \operatorname{tr} R^{4} .
$$


It is sensitive only to the number of gauginos $y$ and hyperinos $s$, which contribute with opposite signs due to their opposite chiralities, and the gravitino and dilatino. The necessary condition $s-y=244$ is fulfilled in our model, as can be seen from Tables A.2 and A.3.

- Quartic non-Abelian anomalies receive contributions from the gaugino in the adjoint representation which need to be cancelled by opposite-chirality hyperinos. Denoting the number of hypermultiplets in representation $\boldsymbol{r}^{i}$ of group factor $G_{A}$ by $s_{A}^{i}$, the quartic terms are

$$
\operatorname{Tr} F_{A}^{4}-\sum_{i} s_{A}^{i} \operatorname{tr}_{\boldsymbol{r}^{i}} F_{A}^{4}, \quad A=\mathrm{SU}(6), \mathrm{SU}(3), \mathrm{SO}(8)
$$

Here $\operatorname{Tr}$ and $\operatorname{tr}_{\boldsymbol{r}^{i}}$ denote traces in the adjoint representation and in the representation $\boldsymbol{r}^{i}$, respectively. We can convert all traces to the fundamental representation (denoted simply by tr), which will introduce representation indices, and possibly terms $\sim\left(\operatorname{tr} F_{A}^{2}\right)^{2}$, and finally leads to the following constraints:

$$
\begin{array}{ll}
\mathrm{SU}(6): & 12+6 s^{\mathbf{2 0}}-s^{\mathbf{6}}-s^{\overline{\mathbf{6}}}=0, \\
\mathrm{SO}(8): & \frac{1}{2} s^{\boldsymbol{8}_{s}}+\frac{1}{2} s^{\boldsymbol{8}_{c}}-s^{\mathbf{8}}=0 .
\end{array}
$$

$\mathrm{SU}(3)$ does not have a fourth-order Casimir invariant and hence $\operatorname{tr} F_{\mathrm{SU}(3)}^{4}$ does not give a condition at this point.

- Finally, the (non-Abelian) $)^{3}$-Abelian anomaly has to vanish for reducibility. Again we convert all traces to the fundamental representation, and have to consider the U(1) charges of the hypermultiplets. We get two nontrivial conditions for each $\mathrm{U}(1)$ ( $\mathrm{SO}(8)$ has no third-order Casimir):

$$
\begin{array}{ll}
\mathrm{SU}(6): & \sum_{\mathbf{6}_{i}} q_{u}^{\mathbf{6}_{i}}-\sum_{\overline{\mathbf{6}}_{i}} q_{u}^{\overline{\mathbf{6}}_{i}}=0, \\
\mathrm{SU}(3): & \sum_{\mathbf{3}_{i}} q_{u}^{\mathbf{3}_{i}}-\sum_{\overline{\mathbf{3}}_{i}} q_{u}^{\overline{\mathbf{3}}_{i}}=0 .
\end{array}
$$

From the U(1) charges in Table A.3 we see that also these constraints are satisfied.

For the remaining anomaly polynomial we normalize the U(1)'s from Table 4.1 by introducing $\hat{t}_{u}=t_{u} / \sqrt{2}\left|t_{u}\right|$. As shown in Appendix B, this leads to a factorization 
of the bulk anomaly polynomial which is of the form (4.5):

$$
\begin{aligned}
\mathrm{i}(2 \pi)^{3} I_{8}^{\mathrm{bulk}}= & \frac{1}{16}\left[\operatorname{tr} R^{2}-2 \operatorname{tr} F_{S U(6)}^{2}-2 \operatorname{tr} F_{S U(3)}^{2}-\operatorname{tr} F_{S O(8)}^{2}-\sum_{u} F_{u}^{2}\right] \\
& \times\left[\operatorname{tr} R^{2}-\sum_{u, v} \beta_{u v} F_{u} F_{v}\right] \\
= & \frac{1}{16} X_{4} Y_{4} .
\end{aligned}
$$

The symmetric coefficient matrix $\beta_{u v}$ in the $\hat{t}_{u}$ basis is

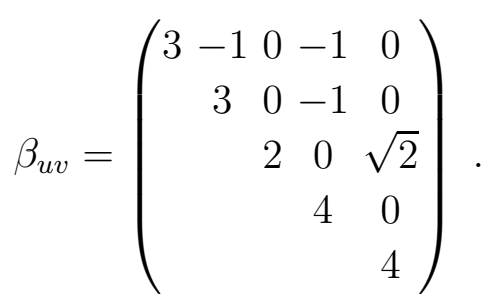

We conclude that all bulk anomalies of our orbifold model are cancelled by variations of the terms $\sim Y_{3}^{(0)}$ in Eq. (4.6).

\subsection{Brane Anomalies}

Since our model contains one Wilson line in the $\mathrm{SO}(4)$ plane, the spectra at the fixed points only depend on $n_{2}$ and not on $n_{2}^{\prime}$, so that we have to evaluate two anomaly polynomials $I_{6}^{0,1}$ in the following.

At a fixed point, there are no gravitational anomalies, and so the only irreducible contributions are non-Abelian cubic ones. Matter now comes in chiral multiplets which can have both chiralities and thus contribute with opposite signs. Furthermore, the anomaly induced by bulk fields surviving the projection is suppressed by a factor of $\frac{1}{4}$ with respect to the contributions from localized fields. Taking this into account, the cubic non-Abelian anomalies are of the form

$$
\frac{1}{4} \sum_{\text {bulk } \mathbf{r}}\left(s_{A}^{(+) \mathbf{r}}-s_{A}^{(-) \mathbf{r}}\right) \operatorname{tr}_{\mathbf{r}} F_{A}^{3}-\sum_{\operatorname{loc} \mathbf{r}} s_{A}^{(-) \mathbf{r}} \operatorname{tr}_{\mathbf{r}} F_{A}^{3},
$$

where the sum is over representations $\mathbf{r}$ of the local group factor $A$, and the $s_{A}^{(+) \mathbf{r}}$ and $s_{A}^{(-) \mathbf{r}}$ denote the number of multiplets in that representation with positive and negative chirality, respectively. We take the localized fields to be left-handed. Using Tables A.4 to A.9, one finds that the model contains no irreducible local anomalies. Vector multiplets do not contribute to anomalies, as they are in a real representation of the gauge group, and neither do the hypermultiplet remnants of $6 \mathrm{D}$ vector multiplets, since they come in left- and right-handed form. 
For the local reducible anomalies we find the following factorization at $n_{2}=0,1$ (cf. Appendix B):

$$
\begin{aligned}
& i(2 \pi)^{3} I_{6}^{0}=-\frac{1}{48}\left[\left(\operatorname{tr} R^{2}\right)\right.-2\left(\operatorname{tr} F_{\mathrm{SU}(5)}^{2}\right)-2\left(\operatorname{tr} F_{\mathrm{SU}(3)}^{2}\right) \\
&-\left.\left(\operatorname{tr} F_{\mathrm{SO}(8)}^{2}\right)-\sum_{u=1}^{6} F_{u}^{2}\right] \times\left(\operatorname{tr}_{0} \hat{t}_{\mathrm{an}}^{0}\right) F^{0}, \\
& i(2 \pi)^{3} I_{6}^{1}=-\frac{1}{48}\left[\left(\operatorname{tr} R^{2}\right)-2\left(\operatorname{tr} F_{\mathrm{SU}(2)}^{2}\right)-2\left(\operatorname{tr} F_{\mathrm{SU}(4)}^{2}\right)-2\left(\operatorname{tr} F_{\mathrm{SU}(2)^{\prime}}^{2}\right)\right. \\
&\left.-2\left(\operatorname{tr} F_{\mathrm{SU}(4)^{\prime}}^{2}\right)-\sum_{v=1}^{8} F_{v}^{2}\right] \times\left(\operatorname{tr}_{1} \hat{t}^{1}\right) F^{1} .
\end{aligned}
$$

The traces of the anomalous U(1)'s are the sums of the charges of the fields present at the given fixed point, and again the contributions of surviving bulk fields are weighted with a factor of $\frac{1}{4}$. The indices $u, v$ in the formulae above run over a basis spanned by the anomalous $\mathrm{U}(1)$ and orthogonal generators, $\hat{t}_{1}^{f} \equiv \hat{t}_{\mathrm{an}}^{f}, \hat{t}_{\mathrm{an}}^{f} \cdot \hat{t}_{u}^{f}=0$, $(u>1)$. The normalization is chosen such that all Abelian factors have level 1 , namely $\hat{t}_{u}^{f}=t_{u}^{f} / \sqrt{2}\left|t_{u}^{f}\right|$. The factorization is of the form (4.5) such that we conclude that all anomalies of our model are cancelled by the localized part of the GreenSchwarz term (4.6).

Equations (4.14) and (4.15) reveal that due to the presence of one Wilson line there are two distinct anomalous $\mathrm{U}(1)$ factors $t_{\mathrm{an}}^{0}$ and $t_{\mathrm{an}}^{1}$ in the model, one for each inequivalent fixed point. For the (unnormalized) anomalous generators from Table 4.1 we find the following traces:

$$
\operatorname{tr}_{0} t_{\mathrm{an}}^{0}=2\left|t_{\mathrm{an}}^{0}\right|^{2}=148, \quad \operatorname{tr}_{1} t_{\mathrm{an}}^{1}=\left|t_{\mathrm{an}}^{1}\right|^{2}=80
$$

The 4D anomalous U(1) follows from integrating the Green-Schwarz term over the internal dimensions. As can be seen from (4.6), this amounts to summing the normalized local U(1)'s. The four-dimensional anomaly polynomial again is of the form (4.5), so we can deduce the anomalous $\mathrm{U}(1)$ in four dimensions from

$$
\frac{\operatorname{tr}_{4 \mathrm{~d}} t_{\mathrm{an}}^{(4 \mathrm{~d})}}{\left|t_{\mathrm{an}}^{(4 \mathrm{~d})}\right|^{2}} t_{\mathrm{an}}^{(4 \mathrm{~d})}=2\left(\frac{\operatorname{tr}_{0} t_{\mathrm{an}}^{0}}{\left|t_{\mathrm{an}}^{0}\right|^{2}} t_{\mathrm{an}}^{0}+\frac{\operatorname{tr}_{1} t_{\mathrm{an}}^{1}}{\left|t_{\mathrm{an}}^{1}\right|^{2}} t_{\mathrm{an}}^{1}\right) .
$$

Here $\operatorname{tr}_{4 \mathrm{~d}}$ denotes the trace over the low-energy spectrum, i.e. zero modes of bulk fields and localized fields, but excluding bulk fields which only survive at $n_{2}=0$ or $n_{2}=1$. Note that the factor of $\frac{1}{4}$ included in the definitions of $\operatorname{tr}_{0}$ and $\operatorname{tr}_{1}$ ensures that zero mode contributions are counted only once. Thus we find the anomalous generator $\hat{t}_{\mathrm{an}}^{(4 \mathrm{~d})}$ from [18] with $\operatorname{tr} t_{\mathrm{an}}^{(4 \mathrm{~d})}=12\left|t_{\mathrm{an}}^{(4 \mathrm{~d})}\right|^{2}=88$ as

$$
t_{\mathrm{an}}^{(4 \mathrm{~d})}=\frac{1}{6}\left(2 t_{\mathrm{an}}^{0}+t_{\mathrm{an}}^{1}\right) .
$$


So all appearing anomalies have been cancelled, either among themselves or by the Green-Schwarz mechanism. We would like to emphasize that there is no free parameter involved: the fields and gauge groups are fixed, as well as the transformation property of $B_{M N}$, which is the only available antisymmetric tensor field which can cancel anomalies. Hence the way in which the different sectors combine in the correct way appears highly non-trivial.

\section{Decoupling of Exotic States}

Let us now consider the decoupling of states with exotic standard model quantum numbers. These are the SU(5) 5-plets of bulk hypermultiplets which originate from the $T_{2} / T_{4}$ - and the untwisted sector, and the $\mathrm{SU}(2)$ doublets and singlets with nonzero hypercharge from the $T_{1} / T_{5}$ - and $T_{3}$-sectors at the fixed points $n_{2}=1$. Note that no exotic matter is located at the fixed points $n_{2}=0$. All the exotic 5-plets and most of the exotic matter at $n_{2}=1$ can be decoupled by VEVs of just a few standard model singlet fields. This decoupling takes place locally at one of the fixed points, which is a crucial difference compared to previous discussions of decoupling in four dimensions $[18,19]$.

The $\mathcal{N}=2$ hypermultiplets $H=\left(H_{L}, H_{R}\right)$ consist of pairs of $\mathcal{N}=1$ leftand right-chiral multiplets either from the $T_{2}$ and $T_{4}$ twisted sectors, or from the untwisted sector. The charge conjugate left-chiral multiplet $H_{R}^{c}$ has the opposite gauge quantum numbers as $H_{L}$. Hence the SU(5) 5- and $\overline{\mathbf{5}}$-hypermultiplets contain the exotic $\mathcal{N}=1$ left-chiral multiplets 5 and $\overline{\mathbf{5}}^{c}$.

The products $\mathbf{5}_{n_{3}} \boldsymbol{5}_{n_{3}}^{c}$ and $\overline{\mathbf{5}}_{n_{3}} \overline{\mathbf{5}}_{n_{3}}^{c}, n_{3}=0,1,2$, are total gauge singlet $\mathcal{N}=1$ chiral multiplets. They do carry, however, non-zero $R$-charges, $R=(-1,-1,0)$ (cf. App. A.1). One easily verifies (cf. Tables A.1, A.4 and A.8) that the product $\bar{Y}_{0}^{c} S_{1} S_{5}$ of standard model singlet fields is a total gauge singlet with $R$-charges $R=(0,0,-1) . S_{1}$ and $S_{5}$ are oscillator states localized at the fixed points $n_{2}=0$. One therefore obtains the local $\mathcal{N}=1$ superpotential terms

$$
W_{1}=\bar{Y}_{0}^{c} S_{1} S_{5}\left(\mathbf{5}_{0} \mathbf{5}_{0}^{c}+\overline{\mathbf{5}}_{0} \overline{\mathbf{5}}_{0}^{c}+\mathbf{5}_{1} \mathbf{5}_{1}^{c}+\overline{\mathbf{5}}_{1} \overline{\mathbf{5}}_{1}^{c}+\mathbf{5}_{2} \mathbf{5}_{2}^{c}+\overline{\mathbf{5}}_{2} \overline{\mathbf{5}}_{2}^{c}\right)
$$

All terms are total gauge singlets with $R$-charges $R=(-1,-1,-1)$. Hence, the H-momentum rules are satisfied, as are the space selection rules (cf. [18]).

From Eq. (5.1) we conclude that a large vacuum expectation value $\left\langle\bar{Y}_{0}^{c} S_{1} S_{5}\right\rangle$ removes 6 pairs of $(\mathbf{5}, \overline{\mathbf{5}})$-plets ${ }^{4}$ from the low energy spectrum. Since we have 3 positive parities for each value of $n_{3}$ (cf. Tables 3.2 and A.3), 6 5- or $\overline{5}$-plets remain. The mass terms are localized at the fixed points $n_{2}=0$. Bulk mass terms between hypermultiplets are forbidden by $\mathcal{N}=2$ supersymmetry.

\footnotetext{
${ }^{4}$ When the distinction between $T_{2^{-}}, T_{4^{-}}$and untwisted sector does not matter, we collectively denote $\mathbf{5}$ and $\overline{\mathbf{5}}^{c}$ by $\mathbf{5}$, and $\overline{\mathbf{5}}$ and $\mathbf{5}^{c}$ by $\overline{\mathbf{5}}$.
} 


\begin{tabular}{|c|ccc|ccccc|}
\hline & $\mathbf{5}$ & $\overline{\mathbf{5}}_{0}^{c}$ & $\mathbf{5}_{1}$ & $\overline{\mathbf{5}}$ & $\mathbf{5}_{0}^{c}$ & $\overline{\mathbf{5}}_{1}$ & $\overline{\mathbf{5}}_{2}$ & $\mathbf{5}_{2}^{c}$ \\
\hline \hline $\mathrm{SU}(3) \times \mathrm{SU}(2)$ & $(1, \mathbf{2})$ & $(\mathbf{3}, 1)$ & $(1, \mathbf{2})$ & $(1, \mathbf{2})$ & $(\overline{\mathbf{3}}, 1)$ & $(1, \mathbf{2})$ & $(\overline{\mathbf{3}}, 1)$ & $(1, \mathbf{2})$ \\
\hline $\mathrm{U}(1)_{B-L}$ & 0 & $-\frac{2}{3}$ & 0 & 0 & $\frac{2}{3}$ & 0 & $-\frac{1}{3}$ & -1 \\
\hline $\mathrm{MSSM}$ & $H_{u}$ & & & & & $H_{d}$ & $d_{3}$ & $l_{3}$ \\
\hline
\end{tabular}

Table 5.1: The remaining $\mathbf{5}$ 's and $\overline{\mathbf{5}}$ 's after the decoupling through $W_{1}$. The $S U(3) \times$ $S U(2)$ representations, $B-L$ charges and MSSM identification refer to the zero modes.

Inspection of Tables 3.2 and A.4 shows that from the $T_{2^{-}}, T_{4^{-}}$and untwisted sectors three $\mathbf{5}$ 's and five $\overline{\mathbf{5}}$ 's remain: $\mathbf{5}, \overline{\mathbf{5}}, \mathbf{5}_{0}^{c}, \overline{\mathbf{5}}_{0}^{c}, \mathbf{5}_{1}, \overline{\mathbf{5}}_{1}, \mathbf{5}_{2}^{c}, \overline{\mathbf{5}}_{2}$. The further decoupling is motivated by phenomenological arguments and by simplicity. The projection condition at the fixed points $n_{2}=1$ leave as $4 \mathrm{D}$ zero modes from each $\mathbf{5}$ and $\overline{\mathbf{5}}$ either an $\mathrm{SU}(3)$ triplet or an $\mathrm{SU}(2)$ doublet. With respect to the $\mathrm{U}(1)_{B-L}$ generator identified in [18],

$$
t_{B-L}=\left(0,1,1,0,0,-\frac{2}{3},-\frac{2}{3},-\frac{2}{3}\right)\left(\frac{1}{2}, \frac{1}{2}, \frac{1}{2},-\frac{1}{2}, 0,0,0,0\right),
$$

these massless states have the $B-L$ charges listed in Table 5.1. This suggests to decouple $\overline{\mathbf{5}}_{0}^{c}$ and $\mathbf{5}_{0}^{c}$, which is possible with a local coupling at the fixed point $n_{2}=0$,

$$
W_{2}=Y_{0} S_{1} S_{5} \mathbf{5}_{0}^{c} \overline{\mathbf{5}}_{0}^{c},
$$

and a large $\operatorname{VEV}\left\langle Y_{0} S_{1} S_{5}\right\rangle$.

From the remaining $\mathbf{5}$-plets, either $\mathbf{5}$ or $\mathbf{5}_{1}$ can be chosen as Higgs field $H_{u}$. A large top-quark coupling is obtained for $\mathbf{5} \supset H_{u} . \mathbf{5}_{1}$ can be easily decoupled using the $6 \mathrm{D}$ gauge coupling with the chiral multiplet $\overline{\mathbf{5}}$ of the $\mathrm{SU}(6) 35$-plet,

$$
W_{H} \supset \sqrt{2} g\left(X_{0} \mathbf{5} \mathbf{5}_{0}^{c}+\bar{X}_{0} \overline{\mathbf{5}}_{0}^{c}+X_{1}^{c} \mathbf{5}_{1} \overline{\mathbf{5}}+\bar{X}_{1}^{c} \overline{\mathbf{5}}_{1} \mathbf{5}+X_{2} \mathbf{5} \mathbf{5}_{2}^{c}+\bar{X}_{2}^{c} \overline{\mathbf{5}}_{2} \mathbf{5}\right),
$$

with a large VEV $\left\langle\bar{X}_{1}^{c}\right\rangle$. The remaining $\overline{\mathbf{5}}$-plets $\mathbf{5}_{2}^{c}$ and $\overline{\mathbf{5}}_{1}$ then correspond to a lepton doublet and the Higgs field $H_{d}$, respectively. The chosen vacuum is similar to the $B-L$ conserving vacuum discussed in [18]. It corresponds to partial gauge-Higgs unification for $H_{u}$. If one chooses to decouple 5 instead of $\boldsymbol{5}_{1}$, one has no gauge-Higgs unification. Alternatively, one can also keep $\mathbf{5}$ and $\overline{\mathbf{5}}$ massless, corresponding to full gauge-Higgs unification.

All other exotic states are localized at $n_{2}=1$. The $\mathrm{SU}(2)$ doublets $M_{i}$ and some of the $\mathrm{SU}(2)$ singlets $S_{i}^{ \pm}$can already be decoupled by cubic terms,

$$
\begin{aligned}
W_{3}= & \bar{Z}_{1}^{c} M_{1} M_{4}+Z_{0}^{c} M_{2} M_{3}, \\
W_{4}= & \bar{Y}_{2}^{c}\left(S_{2}^{+} S_{1}^{-}+S_{3}^{+} S_{4}^{-}\right)+Z_{2}\left(S_{4}^{+} S_{5}^{-}+S_{4}^{+} S_{5}^{\prime-}\right) \\
& +\bar{Z}_{2}\left(S_{3}^{-} S_{6}^{+}+S_{3}^{-} S_{6}^{\prime+}\right)+U_{1}^{c}\left(S_{6}^{+} S_{5}^{-}+S_{6}^{\prime+} S_{5}^{\prime-}\right),
\end{aligned}
$$


with large VEVs $\left\langle\bar{Z}_{1}^{c}\right\rangle,\left\langle Z_{0}^{c}\right\rangle,\left\langle\bar{Y}_{2}^{c}\right\rangle,\left\langle Z_{2}\right\rangle,\left\langle U_{1}^{c}\right\rangle$. The decoupling of the remaining exotic singlets with hypercharge, $S_{1}^{+}, S_{2}^{-}, S_{5}^{+}, S_{6}^{-}, S_{7}^{-}, S_{7}^{+}$requires higher dimensional operators (cf. [18,19]), which we will not discuss further in this paper.

After the decoupling of altogether 8 pairs of $(\mathbf{5}, \overline{\mathbf{5}})$-plets we are left with two localized families,

$$
\left(n_{2}, n_{2}^{\prime}\right)=(0,0): \overline{\mathbf{5}}_{(1)}, \mathbf{1 0}_{(1)} ; \quad\left(n_{2}, n_{2}^{\prime}\right)=(0,1): \overline{\mathbf{5}}_{(2)}, \mathbf{1 0}_{(2)},
$$

together with two further families and a pair of Higgs doublets in the bulk:

$$
\overline{\mathbf{5}}_{(3)} \equiv \mathbf{5}_{2}^{c}, 10_{(3)} \equiv \mathbf{1 0} ; \quad \overline{\mathbf{5}}_{(4)} \equiv \overline{\mathbf{5}}_{2}, \mathbf{1 0}_{(4)} \equiv \overline{\mathbf{1 0}}^{c} ; \quad H_{u} \equiv \mathbf{5}, H_{d} \equiv \overline{\mathbf{5}}_{1} .
$$

At the fixed points $n_{2}=0$ these chiral $\mathcal{N}=1$ multiplets form a local SU(5) GUT theory. The corresponding Yukawa couplings will be discussed in the following section. From the two bulk families, half of the states are projected out by the projection conditions at $n_{2}=1$, and together they give rise to one family of zero modes (cf. Eq. (3.9) and Tab. 5.1).

Note that the decoupling terms (5.1), (5.3), (5.5) and (5.6) require VEVs of both bulk and localized fields. The localized singlets $S_{1}$ and $S_{5}$ correspond to oscillator modes. As we will see in Section 7, bulk and brane field backgrounds are typically induced by local Fayet-Iliopoulos (FI) terms. The non-vanishing VEVs of localized fields are often related to a resolution of the orbifold singularities [31,32]. However, a study of the blow-up of the considered orbifold to a smooth manifold and the geometrical interpretation of the localized VEVs is beyond the scope of this work.

\section{Yukawa Couplings}

In the previous section we have obtained four quark-lepton families transforming as $\left(\overline{\mathbf{5}}_{(i)}+\mathbf{1 0}_{(i)}\right)$ under $\mathrm{SU}(5)$, where $i$ is a generation index. Two families are localized at the branes $(i=1,2)$ and two are bulk fields. The corresponding superpotential reads

$$
W_{\text {Yuk }}=C_{i j}^{(u)} \mathbf{1 0}_{(i)} \mathbf{1 0}_{(j)} H_{u}+C_{i j}^{(d)} \overline{\mathbf{5}}_{(i)} \mathbf{1 0}_{(j)} H_{d},
$$

where the couplings $C_{i j}^{(u)}$ and $C_{i j}^{(u)}$ are composed of singlet fields such that the superpotential obeys the string selection rules (cf. [18]).

As an example, we consider a vacuum where in addition to the fields

$$
\bar{Y}_{0}^{c}, S_{1}, S_{5}, Y_{0}, X_{1}^{c}, \bar{Z}_{1}^{c}, Z_{0}^{c}, \bar{Y}_{2}^{c}, Z_{2}, \bar{Z}_{2}, U_{1}^{c},
$$

used in Section 5 for decoupling, only the singlets

$$
Y_{0}^{c}, Y_{1}, \bar{Y}_{1}, S_{3}, S_{4}, S_{7}
$$


acquire non-zero VEVs. After a straightforward calculation, we find that up to $\mathcal{O}(8)$ in the fields, this vacuum leads to couplings

$$
C_{i j}^{(u)}=\left(\begin{array}{cccc}
a_{1} & 0 & a_{2} & a_{3} \\
0 & a_{1} & a_{2} & a_{3} \\
a_{2} & a_{2} & 0 & g \\
a_{3} & a_{3} & g & a_{4}
\end{array}\right), \quad C_{i j}^{(d)}=\left(\begin{array}{cccc}
0 & 0 & b_{1} & b_{2} \\
0 & 0 & b_{1} & b_{2} \\
b_{3} & b_{3} & b_{4} & 0 \\
b_{5} & b_{5} & b_{6} & b_{5}^{2}
\end{array}\right),
$$

with

$$
\begin{array}{lll}
a_{1}=\left\langle Y_{0}^{c} \bar{Y}_{0}^{c} S_{1} S_{3}\right\rangle, & a_{2}=\left\langle\left(\bar{Y}_{0}^{c} S_{1}\right)^{2} S_{5}\right\rangle, & a_{3}=\left\langle Y_{0}^{c} \bar{Y}_{0}^{c} S_{1} S_{3} S_{5}\right\rangle, \\
a_{4}=\left\langle Y_{0}^{c} \bar{Y}_{0}^{c} S_{1} S_{3}\left(S_{5}\right)^{2}\right\rangle, & & \\
b_{1}=\left\langle Y_{0} \bar{Y}_{1}\left(S_{5}\right)^{3}\left(S_{7}\right)^{2}\right\rangle, & b_{2}=\left\langle X_{1}^{c} \bar{Y}_{2}^{c} U_{1}^{c} S_{7}\right\rangle, & b_{3}=\left\langle X_{1}^{c} \bar{Y}_{1} S_{3}\left(S_{5} S_{7}\right)^{2}\right\rangle, \\
b_{4}=\left\langle\left(X_{1}^{c}\right)^{2} \bar{Y}_{1} U_{1}^{c} S_{4} S_{7}\right\rangle, & b_{5}=\left\langle S_{5}\right\rangle, & b_{6}=\left\langle\left(X_{1}^{c}\right)^{2} Y_{1} S_{1} S_{7}\right\rangle .
\end{array}
$$

Note that the chosen vacuum yields non-vanishing Yukawa couplings while the $\mu$ term is only generated at higher order.

The Yukawa couplings (6.1) are SU(5) invariant, hence we have obtained an SU(5) GUT model. Note that the SU(5) Yukawa interactions are local since the fields $S_{i}$ are localized at the fixed points $n_{2}=0$, i.e., we have a local SU(5) GUT model. The only exception is $C_{34}^{(u)}=C_{43}^{(u)}=g$, which is a remnant of the $\operatorname{SU}(6)$ bulk gauge interaction. It is a consequence of the partial gauge-Higgs unification of the present model, which implies a phenomenologically attractive large top Yukawa coupling.

We can now proceed and deduce the corresponding Yukawa couplings in four dimensions. As described in Section 0 , half of each of the two bulk families is projected out by the additional $\mathbb{Z}_{2}$ orbifold condition at the second pair of fixed points $\left(n_{2}=1\right)$. The remaining fields from the split bulk matter multiplets then form the content of the third standard model family. The 4D Yukawa terms are

$$
W_{\mathrm{Yuk}}=Y_{i j}^{(u)} u_{i}^{c} q_{j} H_{u}+Y_{i j}^{(d)} d_{i}^{c} q_{j} H_{d}+Y_{i j}^{(l)} l_{i} e_{j}^{c} H_{d}
$$

where $i, j=1,2,3$ is a family index, and

$$
Y_{i j}^{(u)}=\left(\begin{array}{ccc}
a_{1} & 0 & a_{3} \\
0 & a_{1} & a_{3} \\
a_{2} & a_{2} & g
\end{array}\right), \quad Y_{i j}^{(d)}=\left(\begin{array}{ccc}
0 & 0 & b_{2} \\
0 & 0 & b_{2} \\
b_{5} & b_{5} & b_{7}
\end{array}\right), \quad Y_{i j}^{(l)}=\left(\begin{array}{ccc}
0 & 0 & b_{1} \\
0 & 0 & b_{1} \\
b_{3} & b_{3} & b_{4}
\end{array}\right) .
$$

The Yukawa matrices for down quarks and leptons are different, although they originate from $\mathrm{SU}(5)$ invariant couplings of the $6 \mathrm{D}$ theory. This is due to the split multiplets which form the third quark-lepton family. In this way the mostly unsuccessful SU(5) predictions for fermion masses are avoided. However, one also loses the successful prediction $m_{b}\left(M_{\mathrm{GUT}}\right) \simeq m_{\tau}\left(M_{\mathrm{GUT}}\right)$. 


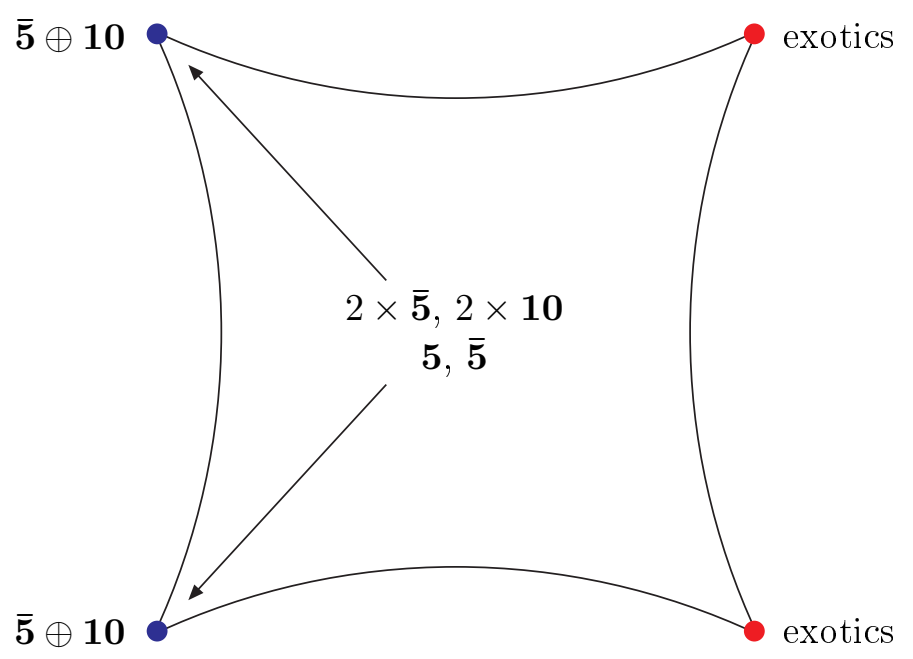

Figure 2: The orbifold $T^{2} / \mathbb{Z}_{2}$. The blue dots (on the left) label the fixed points with $n_{2}=0$, the red ones (right) have $n_{2}=1$. Two quark-lepton generations live at the $n_{2}=0$ fixed points, the third one originates from two $S U(5) \overline{\mathbf{5}}$ and $\mathbf{1 0}$ multiplets in the bulk, half of which is projected out due to the boundary conditions at $n_{2}=1$.

The obtained local SU(5) GUT model is phenomenologically not viable. Not only are electron and down-quark massless, which may be corrected by higher powers of singlet VEVs, but the main problem are $R$-parity violating Yukawa couplings leading to rapid proton decay, which we have not listed. However, the present model is just an example of a large class of models [19], and it is likely that the phenomenology can be improved. In the above discussion we have also ignored neutrino masses which can be generated by a seesaw mechanism typically involving many singlet fields [33].

\section{Supersymmetric Vacua}

In the previous sections we have discussed phenomenologically wanted vacuum configurations, i.e. expectations values of singlet fields, which decouple states with exotic quantum numbers and generate Yukawa couplings for quarks and leptons. The analysis and classification of these vacua is a difficult problem. In particular, one has to show that $\mathcal{N}=1$ supersymmetry remains unbroken in four dimensions. For the present model the conditions for vanishing $F$ - and $D$-terms have been discussed in [18]. A crucial role is played by the Fayet-Iliopoulos $D$-term of the anomalous $\mathrm{U}(1)$, which drives fields away from zero (cf. [34]).

In this paper we are studying the case where two of the compact dimensions are larger than the other four. Such an ansatz assumes that the size of the large dimensions can be stabilized at a scale $1 / M_{\mathrm{GUT}} \gg 1 / M_{\text {string. }}$. To prove this one 
has to find supersymmetric vacua of the effective 6D field theory which incorporates Kaluza-Klein states with masses between $M_{\mathrm{GUT}}$ and $M_{\text {string }}$.

As we saw in Section 4, the 6D theory has different Fayet-Iliopoulos terms at the inequivalent fixed points in the $\mathrm{SO}(4)$-plane (cf. (4.17)),

$$
\mathcal{L}_{\mathrm{FI}}=\sum_{f} \xi_{f} \delta^{2}\left(y-y_{f}\right)\left(-D_{3}^{f}+F_{56}^{f}\right)
$$

where at $f=\left(n_{2}, n_{2}^{\prime}\right)$,

$$
\xi_{(0,0)}=\xi_{(0,1)}=\frac{g M_{\mathrm{P}}^{2}}{384 \pi^{2}} \frac{\operatorname{tr}_{0} t_{\mathrm{an}}^{0}}{\left|t_{\mathrm{an}}^{0}\right|^{2}}, \quad \xi_{(1,0)}=\xi_{(1,1)}=\frac{g M_{\mathrm{P}}^{2}}{384 \pi^{2}} \frac{\operatorname{tr}_{1} t_{\mathrm{an}}^{1}}{\left|t_{\mathrm{an}}^{1}\right|^{2}} .
$$

Integrating over the two compact dimensions reproduces the $4 \mathrm{D}$ Fayet-Iliopoulos term of [18].

In the case of flat space, localized FI terms have been studied in [22], and it has been shown that they lead to an instability of the bulk fields and to spontaneous localization towards the fixed points. For our $6 \mathrm{D}$ supergravity theory this analysis has to be extended to include the gravitational, antisymmetric tensor and dilaton fields. In general, one expects warped solutions, and it is not clear whether $\mathcal{N}=1$ supersymmetry remains unbroken in four dimensions. These questions are beyond the scope of the present paper and will be studied elsewhere.

In the following we will only check whether the VEVs selected in Sections 5 and 6 correspond to a supersymmetric vacuum for an isotropic orbifold, where the $\mathrm{SO}(4)$-, $\mathrm{SU}(3)$ - and $G_{2}$-planes all have string size, and the different FI terms are approximated by a single FI term in four dimensions. As discussed in [18], vanishing $D$-terms are guaranteed if all fields are part of gauge invariant monomials except one which carries negative net anomalous charge. These conditions are indeed satisfied for the vacuum chosen in Sections 5 and 6. Explicit examples of gauge invariant monomials are

$$
X_{3} X_{3}^{c}, X_{4}^{c} S_{1} S_{5}, X_{5}^{c} X_{12}^{c} Y_{1}^{c} Y_{4}^{c} S_{7}^{2}, X_{5}^{c} X_{8} Y_{5} Y_{6} S_{4} S_{7}, X_{5}^{c} X_{8} X_{12}^{c} Z_{1}^{c} S_{3} S_{7}
$$

supplemented by

$$
X_{3}^{c}\left(X_{5}^{c} X_{7}\right)^{2} Y_{8}
$$

which has anomalous charge $-22 / 3$.

Since the superpotential of the standard model singlet fields is unknown, we cannot prove that the $F$-terms vanish for the chosen vacuum. We expect, however, a simplification in the analysis of the superpotential in 6D as compared to $4 \mathrm{D}$, since the superpotential is generated locally at the fixed points where one has larger unbroken symmetries than in the $4 \mathrm{D}$ effective theory. 
It will be very interesting to see whether a supersymmetric vacuum of an isotropic orbifold can be obtained as limiting case from an anisotropic orbifold. The different FI terms at the orbifold fixed points may play a crucial role in generating the anisotropy, and it is intriguing that the mass scale of the FI terms is of the order of the grand unification scale, $M_{\mathrm{P}} / \sqrt{384 \pi^{2}} \sim M_{\mathrm{GUT}}$.

\section{Outlook}

We have constructed a 6D supergravity theory as intermediate step in the compactification of the heterotic string to the supersymmetric standard model in four dimensions. The theory has $\mathcal{N}=2$ supersymmetry and one tensor multiplet, and it has a large number of gravitational, gauge and mixed anomalies, all of which are cancelled by the Green-Schwarz mechanism. The theory is compactified from six to four dimensions on a $\mathbb{Z}_{2}$ orbifold with two inequivalent pairs of fixed points with unbroken $\mathrm{SU}(5)$ and $\mathrm{SU}(2) \times \mathrm{SU}(4)$ symmetry, respectively.

In addition to the cancellation of anomalies, we have been particularly interested in the decoupling of exotic states and the emergence of an intermediate SU(5) GUT. Compared to the $4 \mathrm{D}$ theory the decoupling is more transparent due to the larger symmetries, $\mathcal{N}=2$ supersymmetry in the bulk and larger gauge symmetries at the orbifold fixed points. It is remarkable that most exotic states can be decoupled with VEVs of a few standard model singlet fields at the orbifold fixed points.

A very interesting feature of the theory is the emergence of an intermediate $\mathrm{SU}(5)$ GUT model. Two quark-lepton families are localized at the SU(5) branes and two further families, together with a pair of $\mathbf{5} \oplus \overline{\mathbf{5}}$ plets are bulk fields. SU(5) is broken by the presence of the $\mathrm{SU}(2) \times \mathrm{SU}(4)$ branes. This generates a pair of Higgs doublets as split multiplets. Split multiplets of the two bulk quark-lepton families also form the third quark-lepton family, with the standard model quantum numbers of one $\overline{\mathbf{5}}$-plet and one 10-plet. Due to the presence of the split multiplets, the Yukawa couplings of the $4 \mathrm{D}$ theory break SU(5) explicitly, thus avoiding unsuccessful SU(5) predictions of ordinary 4D GUTs.

The 6D theory originally has a large number of $\mathbf{5} \oplus \overline{\mathbf{5}}$ pairs, most of which are decoupled. As discussed in Section 5, the identification of the Higgs fields depends on the choice of the vacuum configuration, and one can have no, partial or full gaugeHiggs unification. Since there is no clear distinction between matter and Higgs fields, one generically expects large $R$-parity breaking Yukawa couplings leading to fast proton decay, as it is indeed the case for the vacuum chosen in Sections 5 and 6 . However, since the considered model is just one example of a large class of similar models [19], it is likely that the phenomenology can be improved.

On the theoretical side, the main open problems concerns the stabilization of extra dimensions at a scale $1 / M_{\mathrm{GUT}} \gg 1 / M_{\text {string }}$ and the existence of corresponding 
vacua with unbroken $\mathcal{N}=1$ supersymmetry. We hope to address these questions elsewhere.

\section{Acknowlegments}

We would like to thank S. Groot Nibbelink, A. Hebecker, J. Louis and M. Trapletti for valuable discussions. 


\section{A. States}

\section{A.1 $R$-Charges}

The $R$-charges of a chiral multiplet are defined as $R^{i}=q_{\mathrm{sh}}^{i}-\left(\widetilde{N}-\widetilde{N}^{*}\right)^{i}$, where $q_{\mathrm{sh}}^{i}$ is the shifted H-momentum of the scalar and the vectors $\widetilde{N}$ and $\widetilde{N}^{*}$ denote oscillator numbers of left-moving fields in $z^{i}$ and $\bar{z}^{i}$ directions, respectively.

\begin{tabular}{|c|c|c|ccc|}
\hline Sector & State & Excitation & $R^{1}$ & $R^{2}$ & $R^{3}$ \\
\hline \hline$U$ & $U_{1}^{c}$ & & 0 & -1 & 0 \\
$U$ & $U_{2}, U_{3}, U_{4}$ & & -1 & 0 & 0 \\
\hline$T_{1}$ & All & & $-\frac{1}{6}$ & $-\frac{1}{3}$ & $-\frac{1}{2}$ \\
\hline$T_{1}^{*}$ & $S_{1}, S_{2}, S_{7}$ & $\widetilde{N}^{*}=(1,0,0)$ & $\frac{5}{6}$ & $-\frac{1}{3}$ & $-\frac{1}{2}$ \\
$T_{1}^{*}$ & $S_{4}, S_{6}$ & $\widetilde{N}^{*}=(2,0,0)$ & $\frac{11}{6}$ & $-\frac{1}{3}$ & $-\frac{1}{2}$ \\
$T_{1}^{*}$ & $S_{3}, S_{5}$ & $\widetilde{N}^{*}=(0,1,0)$ & $-\frac{1}{6}$ & $\frac{2}{3}$ & $-\frac{1}{2}$ \\
\hline$T_{2}$ & $H_{L}$ & & $-\frac{1}{3}$ & $-\frac{2}{3}$ & 0 \\
\hline$T_{2}^{*}$ & $Y_{n_{3}}^{*}$ & $\widetilde{N}^{\prime}=(0,1,0)$ & $-\frac{1}{3}$ & $-\frac{5}{3}$ & 0 \\
$T_{2}^{*}$ & $Y_{n_{3}}^{\prime}$ & $\widetilde{N}^{*}=(1,0,0)$ & $\frac{2}{3}$ & $-\frac{2}{3}$ & 0 \\
\hline$T_{3}$ & $\mathrm{All}$ & & $-\frac{1}{2}$ & 0 & $-\frac{1}{2}$ \\
\hline$T_{4}$ & $H_{R}^{c}$ & & $-\frac{2}{3}$ & $-\frac{1}{3}$ & 0 \\
\hline$T_{4}^{*}$ & $Y_{n_{3}}^{* c}$ & $\widetilde{N}^{*}=(0,1,0)$ & $-\frac{2}{3}$ & $\frac{2}{3}$ & 0 \\
$T_{4}^{*}$ & $Y_{n_{3}}^{* * c}$ & $\widetilde{N}=(1,0,0)$ & $-\frac{5}{3}$ & $-\frac{1}{3}$ & 0 \\
\hline
\end{tabular}

Table A.1: $R$-charges and oscillator excitations of left-handed states. $U$ denotes the untwisted sector and a star represents non-vanishing oscillator numbers. 


\section{A.2 Bulk States}

Here we list the states of the effective $6 \mathrm{D}$ bulk theory. They are obtained from the heterotic string by an $\mathbb{Z}_{3}$ orbifold projection with one Wilson line, as described in Section 2.

\begin{tabular}{|c|c|c|c|c|c|c|c|}
\hline Multiplet & Representation & $t_{1}$ & $t_{2}$ & $t_{3}$ & $t_{4}$ & $t_{5}$ & $\#$ \\
\hline \hline Graviton & & & & & & & 1 \\
Tensor & & & & & & & 1 \\
Hyper & & & & & & & 2 \\
\hline Vector & $(\mathbf{3 5} ; 1,1)$ & & & & & & 35 \\
& $(1 ; \mathbf{8}, 1)$ & & & & & & 8 \\
& $(1 ; 1, \mathbf{2 8})$ & & & & & & 28 \\
& $5 \times(1 ; 1,1)$ & & & & & & 5 \\
\hline Hyper & $(\mathbf{2 0} ; 1,1)$ & $-\frac{1}{2}$ & $\frac{1}{2}$ & 0 & 0 & 0 & 20 \\
& $(1 ; 1,8)$ & 0 & 0 & 0 & -1 & 0 & 8 \\
& $\left(1 ; 1, \mathbf{8}_{s}\right)$ & 0 & 0 & 0 & $\frac{1}{2}$ & $\frac{3}{2}$ & 8 \\
& $\left(1 ; 1, \mathbf{8}_{c}\right)$ & 0 & 0 & 0 & $\frac{1}{2}$ & $-\frac{3}{2}$ & 8 \\
& $(1 ; 1,1)$ & $\frac{1}{2}$ & $\frac{1}{2}$ & -3 & 0 & 0 & 1 \\
& $(1 ; 1,1)$ & -1 & -1 & 0 & 0 & 0 & 1 \\
& $(1 ; 1,1)$ & 1 & -1 & 0 & 0 & 0 & 1 \\
& $(1 ; 1,1)$ & $\frac{1}{2}$ & $\frac{1}{2}$ & 3 & 0 & 0 & 1 \\
\hline
\end{tabular}

Table A.2: The massless spectrum of the $6 D$ theory arising from the untwisted sector. There are 76 vector multiplets and 50 hypermultiplets. The second column refers to the representations with respect to $S U(6) \times S U(3) \times S O(8), t_{1}-t_{5}$ are the charges with respect to the $U(1)$ factors of the bulk gauge group. The first three multiplets arise from the $10 \mathrm{D}$ gravitational sector and are complete gauge singlets. 


\begin{tabular}{|c|c|c|c|c|c|c|c|c|}
\hline Sector & Representation & $n_{3}$ & $t_{1}$ & $t_{2}$ & $t_{3}$ & $t_{4}$ & $t_{5}$ & $\#$ \\
\hline \multirow[t]{4}{*}{$T_{2} / T_{4}$} & $3 \times(\mathbf{6} ; 1,1)$ & 0 & 0 & $-\frac{1}{3}$ & 1 & $\frac{2}{3}$ & 0 & 18 \\
\hline & $3 \times(\overline{\mathbf{6}} ; 1,1)$ & 0 & 0 & $-\frac{1}{3}$ & -1 & $\frac{2}{3}$ & 0 & 18 \\
\hline & $3 \times(1 ; 1,1)$ & 0 & -1 & $-\frac{1}{3}$ & 0 & $\frac{2}{3}$ & 0 & 3 \\
\hline & $3 \times(1 ; 1,1)$ & 0 & 1 & $-\frac{1}{3}$ & 0 & $\frac{2}{3}$ & 0 & 3 \\
\hline \multirow[t]{3}{*}{$T_{2} / T_{4}$} & $3 \times(1 ; 3,1)$ & 0 & 0 & $\frac{2}{3}$ & 0 & $-\frac{1}{3}$ & 1 & 9 \\
\hline & $3 \times(1 ; \overline{\mathbf{3}}, 1)$ & 0 & 0 & $\frac{2}{3}$ & 0 & $-\frac{1}{3}$ & -1 & 9 \\
\hline & $3 \times(1 ; 1,8)$ & 0 & 0 & $\frac{2}{3}$ & 0 & $-\frac{1}{3}$ & 0 & 24 \\
\hline$T_{2} / T_{4}^{*}$ & $6 \times(1 ; 1,1)$ & 0 & 0 & $\frac{2}{3}$ & 0 & $\frac{2}{3}$ & 0 & 6 \\
\hline \multirow[t]{4}{*}{$T_{2} / T_{4}$} & $3 \times(\mathbf{6} ; 1,1)$ & 1 & 0 & $-\frac{1}{3}$ & -1 & $-\frac{1}{3}$ & -1 & 18 \\
\hline & $3 \times(\overline{\mathbf{6}} ; 1,1)$ & 1 & $\frac{1}{2}$ & $\frac{1}{6}$ & 0 & $-\frac{1}{3}$ & -1 & 18 \\
\hline & $3 \times(1 ; 1,1)$ & 1 & 0 & $\frac{2}{3}$ & -2 & $-\frac{1}{3}$ & -1 & 3 \\
\hline & $3 \times(1 ; 1,1)$ & 1 & $\frac{1}{2}$ & $-\frac{5}{6}$ & 1 & $-1 \frac{1}{3}$ & -1 & 3 \\
\hline \multirow[t]{3}{*}{$T_{2} / T_{4}$} & $3 \times(1 ; \mathbf{3}, 1)$ & 1 & $-\frac{1}{2}$ & $\frac{1}{6}$ & 1 & $\frac{2}{3}$ & 0 & 9 \\
\hline & $3 \times(1 ; \overline{\mathbf{3}}, 1)$ & 1 & $-\frac{1}{2}$ & $\frac{1}{6}$ & 1 & $-\frac{1}{3}$ & 1 & 9 \\
\hline & $3 \times\left(1 ; 1, \mathbf{8}_{s}\right)$ & 1 & $-\frac{1}{2}$ & $\frac{1}{6}$ & 1 & $\frac{1}{6}$ & $\frac{1}{2}$ & 24 \\
\hline$T_{2} / T_{4}{ }^{*}$ & $6 \times(1 ; 1,1)$ & 1 & $-\frac{1}{2}$ & $\frac{1}{6}$ & 1 & $-\frac{1}{3}$ & -1 & 6 \\
\hline \multirow[t]{4}{*}{$T_{2} / T_{4}$} & $3 \times(\mathbf{6} ; 1,1)$ & 2 & $\frac{1}{2}$ & $\frac{1}{6}$ & 0 & $-\frac{1}{3}$ & 1 & 18 \\
\hline & $3 \times(\overline{\mathbf{6}} ; 1,1)$ & 2 & 0 & $-\frac{1}{3}$ & 1 & $-\frac{1}{3}$ & 1 & 18 \\
\hline & $3 \times(1 ; 1,1)$ & 2 & $\frac{1}{2}$ & $-\frac{5}{6}$ & -1 & $-\frac{1}{3}$ & 1 & 3 \\
\hline & $3 \times(1 ; 1,1)$ & 2 & 0 & $\frac{2}{3}$ & 2 & $-\frac{1}{3}$ & 1 & 3 \\
\hline \multirow[t]{3}{*}{$T_{2} / T_{4}$} & $3 \times(1 ; 3,1)$ & 2 & $-\frac{1}{2}$ & $\frac{1}{6}$ & -1 & $-\frac{1}{3}$ & -1 & 9 \\
\hline & $3 \times(1 ; \overline{\mathbf{3}}, 1)$ & 2 & $-\frac{1}{2}$ & $\frac{1}{6}$ & -1 & $\frac{2}{3}$ & 0 & 9 \\
\hline & $3 \times\left(1 ; 1,8_{c}\right)$ & 2 & $-\frac{1}{2}$ & $\frac{1}{6}$ & -1 & $\frac{1}{6}$ & $-\frac{1}{2}$ & 24 \\
\hline$T_{2} / T_{4}{ }^{*}$ & $6 \times(1 ; 1,1)$ & 2 & $-\frac{1}{2}$ & $\frac{1}{6}$ & -1 & $-\frac{1}{3}$ & 1 & 6 \\
\hline
\end{tabular}

Table A.3: The massless spectrum of the $6 D$ theory arising from the $T_{2}$ and $T_{4}$ sectors. There are 270 hypermultiplets. The states are localised in the $G_{2}$ and $S U(3)$ planes, which contain three fixed points each. The equivalent $G_{2}$ fixed points yield the multiplicity factor three, localization in the $S U(3)$ plane is given by $n_{3} . T_{2} / T_{4}{ }^{*}$ states have non-vanishing oscillator numbers. 


\section{A.3 States at the Fixed Points}

Here we list the states at the fixed points $n_{2}=0,1$. These involve bulk states from the $T_{2} / T_{4}$ and the untwisted sector (see Tables 3.2 - 3.5) and localized states from the sectors $T_{1} / T_{5}$ and $T_{3} . X_{i}, \bar{X}_{i}, Y_{i}, \bar{Y}_{i}, Z_{i}, \bar{Z}_{i}$ and $U_{i}$ are bulk fields; $S_{1}-S_{8}$ are localized fields.

\begin{tabular}{|c||c|c||c|c||c|c|c|c|c|c||c|}
\hline Bulk & $n_{2}=0$ & $n_{3}$ & $H_{L}$ & $H_{R}$ & $t_{6}^{0}$ & $t_{1}$ & $t_{2}$ & $t_{3}$ & $t_{4}$ & $t_{5}$ & \\
\hline \hline$(\mathbf{6} ; 1,1)$ & $(\mathbf{5} ; 1,1)$ & 0 &,,-+- &,,+-+ & -1 & 0 & $-\frac{1}{3}$ & 1 & $\frac{2}{3}$ & 0 & \\
& $(1 ; 1,1)$ & 0 &,,+-+ &,,-+- & 5 & 0 & $-\frac{1}{3}$ & 1 & $\frac{2}{3}$ & 0 & $X_{0}$ \\
\hline$(\overline{\mathbf{6}} ; 1,1)$ & $(\overline{\mathbf{5}} ; 1,1)$ & 0 &,,-+- &,,+-+ & 1 & 0 & $-\frac{1}{3}$ & -1 & $\frac{2}{3}$ & 0 & \\
& $(1 ; 1,1)$ & 0 &,,+-+ &,,-+- & -5 & 0 & $-\frac{1}{3}$ & -1 & $\frac{2}{3}$ & 0 & $\bar{X}_{0}$ \\
\hline$(1 ; 1,1)$ & $(1 ; 1,1)$ & 0 &,,+-+ &,,-+- & 0 & 1 & $-\frac{1}{3}$ & 0 & $\frac{2}{3}$ & 0 & $Y_{0}$ \\
\hline$(1 ; 1,1)$ & $(1 ; 1,1)$ & 0 &,,+-+ &,,-+- & 0 & -1 & $-\frac{1}{3}$ & 0 & $\frac{2}{3}$ & 0 & $\bar{Y}_{0}$ \\
\hline \hline$(1 ; \mathbf{3}, 1)$ & $(1 ; \mathbf{3}, 1)$ & 0 &,,-+- &,,+-+ & 0 & 0 & $\frac{2}{3}$ & 0 & $-\frac{1}{3}$ & 1 & \\
\hline$(1 ; \overline{\mathbf{3}}, 1)$ & $(1 ; \overline{\mathbf{3}}, 1)$ & 0 &,,-+- &,,+-+ & 0 & 0 & $\frac{2}{3}$ & 0 & $-\frac{1}{3}$ & -1 & \\
\hline$(1 ; 1,8)$ & $(1 ; 1,8)$ & 0 &,,-+- &,,+-+ & 0 & 0 & $\frac{2}{3}$ & 0 & $-\frac{1}{3}$ & 0 & \\
\hline \hline$(\mathbf{6} ; 1,1)$ & $(\mathbf{5} ; 1,1)$ & 1 &,,+-+ &,,-+- & -1 & 0 & $-\frac{1}{3}$ & -1 & $-\frac{1}{3}$ & -1 & \\
& $(1 ; 1,1)$ & 1 &,,-+- &,,+-+ & 5 & 0 & $-\frac{1}{3}$ & -1 & $-\frac{1}{3}$ & -1 & $X_{1}$ \\
\hline$(\overline{\mathbf{6}} ; 1,1)$ & $(\overline{\mathbf{5}} ; 1,1)$ & 1 &,,+-+ &,,-+- & 1 & $\frac{1}{2}$ & $\frac{1}{6}$ & 0 & $-\frac{1}{3}$ & -1 & \\
& $(1 ; 1,1)$ & 1 &,,-+- &,,+-+ & -5 & $\frac{1}{2}$ & $\frac{1}{6}$ & 0 & $-\frac{1}{3}$ & -1 & $\bar{X}_{1}$ \\
\hline$(1 ; 1,1)$ & $(1 ; 1,1)$ & 1 &,,+-+ &,,-+- & 0 & 0 & $\frac{2}{3}$ & -2 & $-\frac{1}{3}$ & -1 & $Y_{1}$ \\
\hline$(1 ; 1,1)$ & $(1 ; 1,1)$ & 1 &,,+-+ &,,-+- & 0 & $\frac{1}{2}$ & $-\frac{5}{6}$ & 1 & $-\frac{1}{3}$ & -1 & $\bar{Y}_{1}$ \\
\hline \hline$(1 ; \mathbf{3}, 1)$ & $(1 ; \mathbf{3}, 1)$ & 1 &,,-+- &,,+-+ & 0 & $-\frac{1}{2}$ & $\frac{1}{6}$ & 1 & $\frac{2}{3}$ & 0 & \\
\hline$(1 ; \overline{\mathbf{3}}, 1)$ & $(1 ; \overline{\mathbf{3}}, 1)$ & 1 &,,-+- &,,+-+ & 0 & $-\frac{1}{2}$ & $\frac{1}{6}$ & 1 & $-\frac{1}{3}$ & 1 & \\
\hline$\left(1 ; 1, \mathbf{8}_{\mathbf{s}}\right)$ & $\left(1 ; 1, \mathbf{8}_{\mathbf{s}}\right)$ & 1 &,,+-+ &,,-+- & 0 & $-\frac{1}{2}$ & $\frac{1}{6}$ & 1 & $\frac{1}{6}$ & $\frac{1}{2}$ & \\
\hline \hline$(\mathbf{6} ; 1,1)$ & $(\mathbf{5} ; 1,1)$ & 2 &,,-+- &,,+-+ & -1 & $\frac{1}{2}$ & $\frac{1}{6}$ & 0 & $-\frac{1}{3}$ & 1 & \\
& $(1 ; 1,1)$ & 2 &,,+-+ &,,-+- & 5 & $\frac{1}{2}$ & $\frac{1}{6}$ & 0 & $-\frac{1}{3}$ & 1 & $X_{2}$ \\
\hline$(\overline{\mathbf{6}} ; 1,1)$ & $(\overline{\mathbf{5}} ; 1,1)$ & 2 &,,+-+ &,,-+- & 1 & 0 & $-\frac{1}{3}$ & 1 & $-\frac{1}{3}$ & 1 & \\
& $(1 ; 1,1)$ & 2 &,,,-+- &,,+-+ & -5 & 0 & $-\frac{1}{3}$ & 1 & $-\frac{1}{3}$ & 1 & $\bar{X}_{2}$ \\
\hline$(1 ; 1,1)$ & $(1 ; 1,1)$ & 2 &,,+-+ &,,-+- & 0 & 0 & $\frac{2}{3}$ & 2 & $-\frac{1}{3}$ & 1 & $Y_{2}$ \\
\hline$(1 ; 1,1)$ & $(1 ; 1,1)$ & 2 &,,-+- &,,+-+ & 0 & $\frac{1}{2}$ & $-\frac{5}{6}$ & -1 & $-\frac{1}{3}$ & 1 & $\bar{Y}_{2}$ \\
\hline
\end{tabular}

Table A.4 - continued on next page 
Table A.4 - continued from previous page

\begin{tabular}{|c||c|c||c|c||c|c|c|c|c|c||c|}
\hline Bulk & $n_{2}=0$ & $n_{3}$ & $H_{L}$ & $H_{R}$ & $t_{6}^{0}$ & $t_{1}$ & $t_{2}$ & $t_{3}$ & $t_{4}$ & $t_{5}$ & \\
\hline \hline$(1 ; \mathbf{3}, 1)$ & $(1 ; \mathbf{3}, 1)$ & 2 &,,+-+ &,,-+- & 0 & $-\frac{1}{2}$ & $\frac{1}{6}$ & -1 & $-\frac{1}{3}$ & -1 & \\
\hline$(1 ; \overline{\mathbf{3}}, 1)$ & $(1 ; \overline{\mathbf{3}}, 1)$ & 2 &,,+-+ &,,-+- & 0 & $-\frac{1}{2}$ & $\frac{1}{6}$ & -1 & $\frac{2}{3}$ & 0 & \\
\hline$\left(1 ; 1, \mathbf{8}_{\mathbf{c}}\right)$ & $\left(1 ; 1, \mathbf{8}_{\mathbf{c}}\right)$ & 2 &,,-+- &,,+-+ & 0 & $-\frac{1}{2}$ & $\frac{1}{6}$ & -1 & $\frac{1}{6}$ & $-\frac{1}{2}$ & \\
\hline
\end{tabular}

Table A.4: Local decomposition of ground states from the $T_{2} / T_{4}$ sector at $n_{2}=0$.

The three parities for chiral hypermultiplet components $H_{L}, H_{R}$ correspond to to $q_{\gamma}=0, \frac{1}{2}, 1$.

\begin{tabular}{|c||c|c||c|c||c|c|c|c|c|c|c|c||c|}
\hline Bulk & $n_{2}=1$ & $n_{3}$ & $H_{L}$ & $H_{R}$ & $t_{6}^{1}$ & $t_{7}$ & $t_{8}$ & $t_{1}$ & $t_{2}$ & $t_{3}$ & $t_{4}$ & $t_{5}$ & \\
\hline \hline$(\mathbf{6} ; 1,1)$ & $(1, \mathbf{4} ; 1,1)$ & 0 &,,-+- &,,+-+ & 5 & 0 & 0 & 0 & $-\frac{1}{3}$ & 1 & $\frac{2}{3}$ & 0 & \\
& $(\mathbf{2}, 1 ; 1,1)$ & 0 &,,+-+ &,,-+- & -10 & 0 & 0 & 0 & $-\frac{1}{3}$ & 1 & $\frac{2}{3}$ & 0 & \\
\hline$(\overline{\mathbf{6}} ; 1,1)$ & $(1, \overline{\mathbf{4}} ; 1,1)$ & 0 &,,-+- &,,+-+ & -5 & 0 & 0 & 0 & $-\frac{1}{3}$ & -1 & $\frac{2}{3}$ & 0 & \\
& $(\mathbf{2}, 1 ; 1,1)$ & 0 &,,+-+ &,,-+- & 10 & 0 & 0 & 0 & $-\frac{1}{3}$ & -1 & $\frac{2}{3}$ & 0 & \\
\hline$(1 ; 1,1)$ & $(1,1 ; 1,1)$ & 0 &,,+-+ &,,-+- & 0 & 0 & 0 & 1 & $-\frac{1}{3}$ & 0 & $\frac{2}{3}$ & 0 & $Y_{0}$ \\
\hline$(1 ; 1,1)$ & $(1,1 ; 1,1)$ & 0 &,,+-+ &,,-+- & 0 & 0 & 0 & -1 & $-\frac{1}{3}$ & 0 & $\frac{2}{3}$ & 0 & $\bar{Y}_{0}$ \\
\hline \hline$(1 ; \mathbf{3}, 1)$ & $(1,1 ; \mathbf{2}, 1)$ & 0 &,,+-+ &,,-+- & 0 & 1 & 0 & 0 & $\frac{2}{3}$ & 0 & $-\frac{1}{3}$ & 1 & \\
& $(1,1 ; 1,1)$ & 0 &,,-+- &,,+-+ & 0 & -2 & 0 & 0 & $\frac{2}{3}$ & 0 & $-\frac{1}{3}$ & 1 & $Z_{0}$ \\
\hline$(1 ; \overline{\mathbf{3}}, 1)$ & $(1,1 ; \mathbf{2}, 1)$ & 0 &,,-+- &,,+-+ & 0 & -1 & 0 & 0 & $\frac{2}{3}$ & 0 & $-\frac{1}{3}$ & -1 & \\
& $(1,1 ; 1,1)$ & 0 &,,+-+ &,,-+- & 0 & 2 & 0 & 0 & $\frac{2}{3}$ & 0 & $-\frac{1}{3}$ & -1 & $Z_{0}$ \\
\hline$(1 ; 1, \mathbf{8})$ & $(1,1 ; 1, \mathbf{4})$ & 0 &,,+-+ &,,-+- & 0 & 0 & -1 & 0 & $\frac{2}{3}$ & 0 & $-\frac{1}{3}$ & 0 & \\
& $(1,1 ; 1, \overline{\mathbf{4}})$ & 0 &,,-+- &,,+-+ & 0 & 0 & 1 & 0 & $\frac{2}{3}$ & 0 & $-\frac{1}{3}$ & 0 & \\
\hline \hline$(\mathbf{6} ; 1,1)$ & $(1, \mathbf{4} ; 1,1)$ & 1 &,,-+- &,,+-+ & 5 & 0 & 0 & 0 & $-\frac{1}{3}$ & -1 & $-\frac{1}{3}$ & -1 & \\
& $(\mathbf{2}, 1 ; 1,1)$ & 1 &,,+-+ &,,-+- & -10 & 0 & 0 & 0 & $-\frac{1}{3}$ & -1 & $-\frac{1}{3}$ & -1 & \\
\hline \hline$(\overline{\mathbf{6}} ; 1,1)$ & $(1, \overline{\mathbf{4}} ; 1,1)$ & 1 &,,-+- &,,+-+ & -5 & 0 & 0 & $\frac{1}{2}$ & $\frac{1}{6}$ & 0 & $-\frac{1}{3}$ & -1 & \\
& $(\mathbf{2}, 1 ; 1,1)$ & 1 &,,+-+ &,,-+- & 10 & 0 & 0 & $\frac{1}{2}$ & $\frac{1}{6}$ & 0 & $-\frac{1}{3}$ & -1 & \\
\hline$(1 ; 1,1)$ & $(1,1 ; 1,1)$ & 1 &,,+-+ &,,-+- & 0 & 0 & 0 & 0 & $\frac{2}{3}$ & -2 & $-\frac{1}{3}$ & -1 & $Y_{1}$ \\
\hline$(1 ; 1,1)$ & $(1,1 ; 1,1)$ & 1 &,,+-+ &,,-+- & 0 & 0 & 0 & $\frac{1}{2}$ & $-\frac{5}{6}$ & 1 & $-\frac{1}{3}$ & -1 & $Y_{1}$ \\
\hline$(1 ; \mathbf{3}, 1)$ & $(1,1 ; \mathbf{2}, 1)$ & 1 &,,-+- &,,+-+ & 0 & 1 & 0 & $-\frac{1}{2}$ & $\frac{1}{6}$ & 1 & $\frac{2}{3}$ & 0 & \\
& $(1,1 ; 1,1)$ & 1 &,,+-+ &,,-+- & 0 & -2 & 0 & $-\frac{1}{2}$ & $\frac{1}{6}$ & 1 & $\frac{2}{3}$ & 0 & $Z_{1}$ \\
\hline
\end{tabular}

Table A.5 - continued on next page 
Table A.5 - continued from previous page

\begin{tabular}{|c||c|c||c|c||c|c|c|c|c|c|c|c||c|}
\hline Bulk & $n_{2}=1$ & $n_{3}$ & $H_{L}$ & $H_{R}$ & $t_{6}^{1}$ & $t_{7}$ & $t_{8}$ & $t_{1}$ & $t_{2}$ & $t_{3}$ & $t_{4}$ & $t_{5}$ & \\
\hline \hline$(1 ; \overline{\mathbf{3}}, 1)$ & $(1,1 ; \mathbf{2}, 1)$ & 1 &,,+-+ &,,-+- & 0 & -1 & 0 & $-\frac{1}{2}$ & $\frac{1}{6}$ & 1 & $-\frac{1}{3}$ & 1 & \\
& $(1,1 ; 1,1)$ & 1 &,,-+- &,,+-+ & 0 & 2 & 0 & $-\frac{1}{2}$ & $\frac{1}{6}$ & 1 & $-\frac{1}{3}$ & 1 & $\bar{Z}_{1}$ \\
\hline$\left(1 ; 1, \mathbf{8}_{\mathbf{s}}\right)$ & $(1,1 ; 1, \mathbf{4})$ & 1 &,,+-+ &,,-+- & 0 & 0 & 1 & $-\frac{1}{2}$ & $\frac{1}{6}$ & 1 & $\frac{1}{6}$ & $\frac{1}{2}$ & \\
& $(1,1 ; 1, \overline{\mathbf{4}})$ & 1 &,,-+- &,,+-+ & 0 & 0 & -1 & $-\frac{1}{2}$ & $\frac{1}{6}$ & 1 & $\frac{1}{6}$ & $\frac{1}{2}$ & \\
\hline \hline$(\mathbf{6} ; 1,1)$ & $(1, \mathbf{4} ; 1,1)$ & 2 &,,+-+ &,,-+- & 5 & 0 & 0 & $\frac{1}{2}$ & $\frac{1}{6}$ & 0 & $-\frac{1}{3}$ & 1 & \\
& $(\mathbf{2}, 1 ; 1,1)$ & 2 &,,-+- &,,+-+ & -10 & 0 & 0 & $\frac{1}{2}$ & $\frac{1}{6}$ & 0 & $-\frac{1}{3}$ & 1 & \\
\hline$(\overline{\mathbf{6}} ; 1,1)$ & $(1, \overline{\mathbf{4}} ; 1,1)$ & 2 &,,+-+ &,,-+- & -5 & 0 & 0 & 0 & $-\frac{1}{3}$ & 1 & $-\frac{1}{3}$ & 1 & \\
& $(\mathbf{2}, 1 ; 1,1)$ & 2 &,,-+- &,,+-+ & 10 & 0 & 0 & 0 & $-\frac{1}{3}$ & 1 & $-\frac{1}{3}$ & 1 & \\
\hline$(1 ; 1,1)$ & $(1,1 ; 1,1)$ & 2 &,,-+- &,,+-+ & 0 & 0 & 0 & 0 & $\frac{2}{3}$ & 2 & $-\frac{1}{3}$ & 1 & $Y_{2}$ \\
\hline$(1 ; 1,1)$ & $(1,1 ; 1,1)$ & 2 &,,-+- &,,+-+ & 0 & 0 & 0 & $\frac{1}{2}$ & $-\frac{5}{6}$ & -1 & $-\frac{1}{3}$ & 1 & $\bar{Y}_{2}$ \\
\hline \hline$(1 ; \mathbf{3}, 1)$ & $(1,1 ; \mathbf{2}, 1)$ & 2 &,,-+- &,,+-+ & 0 & 1 & 0 & $-\frac{1}{2}$ & $\frac{1}{6}$ & -1 & $-\frac{1}{3}$ & -1 & \\
& $(1,1 ; 1,1)$ & 2 &,,+-+ &,,-+- & 0 & -2 & 0 & $-\frac{1}{2}$ & $\frac{1}{6}$ & -1 & $-\frac{1}{3}$ & -1 & $Z_{2}$ \\
\hline$(1 ; \overline{\mathbf{3}}, 1)$ & $(1,1 ; \mathbf{2}, 1)$ & 2 &,,-+- &,,+-+ & 0 & -1 & 0 & $-\frac{1}{2}$ & $\frac{1}{6}$ & -1 & $\frac{2}{3}$ & 0 & \\
& $(1,1 ; 1,1)$ & 2 &,,+-+ &,,-+- & 0 & 2 & 0 & $-\frac{1}{2}$ & $\frac{1}{6}$ & -1 & $\frac{2}{3}$ & 0 & $\bar{Z}_{2}$ \\
\hline$\left(1 ; 1, \mathbf{8}_{\mathbf{c}}\right)$ & $(1,1 ; 1, \mathbf{6})$ & 2 &,,-+- &,,+-+ & 0 & 0 & 0 & $-\frac{1}{2}$ & $\frac{1}{6}$ & -1 & $\frac{1}{6}$ & $-\frac{1}{2}$ & \\
& $(1,1 ; 1,1)$ & 2 &,,+-+ &,,-+- & 0 & 0 & 2 & $-\frac{1}{2}$ & $\frac{1}{6}$ & -1 & $\frac{1}{6}$ & $-\frac{1}{2}$ & $Z_{2}^{\prime}$ \\
& $(1,1 ; 1,1)$ & 2 &,,+-+ &,,-+- & 0 & 0 & -2 & $-\frac{1}{2}$ & $\frac{1}{6}$ & -1 & $\frac{1}{6}$ & $-\frac{1}{2}$ & $\bar{Z}_{2}^{\prime}$ \\
\hline
\end{tabular}

Table A.5: Local decomposition of states from the $T_{2} / T_{4}$ sector at $n_{2}=1$. The three parities for chiral hypermultiplet components $H_{L}, H_{R}$ correspond to to $q_{\gamma}=0, \frac{1}{2}, 1$. 


\begin{tabular}{|c||c|c||c|c||c|c|c|c|c|c||c|}
\hline Bulk & $n_{2}=0$ & $n_{3}$ & $H_{L}$ & $H_{R}$ & $t_{6}^{0}$ & $t_{1}$ & $t_{2}$ & $t_{3}$ & $t_{4}$ & $t_{5}$ & \\
\hline$(1 ; 1,1)$ & $(1 ; 1,1)$ & 0 &,,-+- &,,+-+ & 0 & 0 & $\frac{2}{3}$ & 0 & $\frac{2}{3}$ & 0 & $Y_{0}^{*}$ \\
$(1 ; 1,1)$ & $(1 ; 1,1)$ & 0 &,,+-+ &,,-+- & 0 & 0 & $\frac{2}{3}$ & 0 & $\frac{2}{3}$ & 0 & $Y_{0}^{\prime *}$ \\
\hline$(1 ; 1,1)$ & $(1 ; 1,1)$ & 1 &,,-+- &,,+-+ & 0 & $-\frac{1}{2}$ & $\frac{1}{6}$ & 1 & $-\frac{1}{3}$ & -1 & $Y_{1}^{*}$ \\
$(1 ; 1,1)$ & $(1 ; 1,1)$ & 1 &,,+-+ &,,-+- & 0 & $-\frac{1}{2}$ & $\frac{1}{6}$ & 1 & $-\frac{1}{3}$ & -1 & $Y_{1}^{\prime *}$ \\
\hline$(1 ; 1,1)$ & $(1 ; 1,1)$ & 2 &,,+-+ &,,-+- & 0 & $-\frac{1}{2}$ & $\frac{1}{6}$ & -1 & $-\frac{1}{3}$ & 1 & $Y_{2}^{*}$ \\
$(1 ; 1,1)$ & $(1 ; 1,1)$ & 2 &,,-+- &,,+-+ & 0 & $-\frac{1}{2}$ & $\frac{1}{6}$ & -1 & $-\frac{1}{3}$ & 1 & $Y_{2}^{\prime *}$ \\
\hline
\end{tabular}

Table A.6: Local decomposition of excited states from the $T_{2} / T_{4}^{*}$ sector at $n_{2}=0$. The three parities for chiral hypermultiplet components $H_{L}, H_{R}$ correspond to to $q_{\gamma}=0, \frac{1}{2}, 1$. The singlets $Y_{n_{3}}^{*}$ have oscillator numbers $\widetilde{N}=(0,1,0)$, the $Y_{n_{3}}^{\prime *}$ have $\widetilde{N}^{*}=(1,0,0)$.

\begin{tabular}{|c||c|c||c|c||c|c|c|c|c|c|c|c||c|}
\hline Bulk & $n_{2}=1$ & $n_{3}$ & $H_{L}$ & $H_{R}$ & $t_{6}^{1}$ & $t_{7}$ & $t_{8}$ & $t_{1}$ & $t_{2}$ & $t_{3}$ & $t_{4}$ & $t_{5}$ & \\
\hline$(1 ; 1,1)$ & $(1 ; 1,1)$ & 0 &,,+-+ &,,-+- & 0 & 0 & 0 & 0 & $\frac{2}{3}$ & 0 & $\frac{2}{3}$ & 0 & $Y_{0}^{*}$ \\
$(1 ; 1,1)$ & $(1 ; 1,1)$ & 0 &,,-+- &,,+-+ & 0 & 0 & 0 & 0 & $\frac{2}{3}$ & 0 & $\frac{2}{3}$ & 0 & $Y_{0}^{\prime *}$ \\
\hline$(1 ; 1,1)$ & $(1 ; 1,1)$ & 1 &,,+-+ &,,-+- & 0 & 0 & 0 & $-\frac{1}{2}$ & $\frac{1}{6}$ & 1 & $-\frac{1}{3}$ & -1 & $Y_{1}^{*}$ \\
$(1 ; 1,1)$ & $(1 ; 1,1)$ & 1 &,,-+- &,,+-+ & 0 & 0 & 0 & $-\frac{1}{2}$ & $\frac{1}{6}$ & 1 & $-\frac{1}{3}$ & -1 & $Y_{1}^{\prime *}$ \\
\hline$(1 ; 1,1)$ & $(1 ; 1,1)$ & 2 &,,-+- &,,+-+ & 0 & 0 & 0 & $-\frac{1}{2}$ & $\frac{1}{6}$ & -1 & $-\frac{1}{3}$ & 1 & $Y_{2}^{*}$ \\
$(1 ; 1,1)$ & $(1 ; 1,1)$ & 2 &,,+-+ &,,-+- & 0 & 0 & 0 & $-\frac{1}{2}$ & $\frac{1}{6}$ & -1 & $-\frac{1}{3}$ & 1 & $Y_{2}^{\prime *}$ \\
\hline
\end{tabular}

Table A.7: Local decomposition of excited states from the $T_{2} / T_{4}^{*}$ sector at $n_{2}=1$. The three parities for chiral hypermultiplet components $H_{L}, H_{R}$ correspond to to $q_{\gamma}=0, \frac{1}{2}, 1$. The singlets $Y_{n_{3}}^{*}$ have oscillator numbers $\widetilde{N}=(0,1,0)$, the $Y_{n_{3}}^{*}$ have $\widetilde{N}^{*}=(1,0,0)$. 


\begin{tabular}{|c||c|c|c||c|c|c|c|c|c||c|}
\hline Sector & $n_{2}=0$ & $n_{3}$ & $q_{\gamma}$ & $t_{6}^{0}$ & $t_{1}$ & $t_{2}$ & $t_{3}$ & $t_{4}$ & $t_{5}$ & \\
\hline \hline$T_{1} / T_{5}$ & $(\mathbf{1 0} ; 1,1)$ & 0 & $*$ & $\frac{1}{2}$ & 0 & $-\frac{1}{6}$ & $-\frac{1}{2}$ & $\frac{1}{3}$ & 0 & \\
& $(\overline{\mathbf{5}} ; 1,1)$ & 0 & $*$ & $-\frac{3}{2}$ & 0 & $-\frac{1}{6}$ & $\frac{3}{2}$ & $\frac{1}{3}$ & 0 & \\
& $(1 ; 1,1)$ & 0 & $*$ & $\frac{5}{2}$ & 0 & $-\frac{1}{6}$ & $-\frac{5}{2}$ & $\frac{1}{3}$ & 0 & \\
\hline \hline$T_{1} / T_{5}$ & $\left(1 ; 1, \mathbf{8}_{\mathbf{c}}\right)$ & 1 & $*$ & $\frac{5}{2}$ & 0 & $-\frac{1}{6}$ & $-\frac{1}{2}$ & $-\frac{1}{6}$ & $-\frac{1}{2}$ & \\
& $(1 ; \mathbf{3}, 1)$ & 2 & $*$ & $\frac{5}{2}$ & 0 & $-\frac{1}{6}$ & $\frac{3}{2}$ & $\frac{1}{3}$ & 0 & \\
& $(1 ; 1,1)$ & 2 & $*$ & $\frac{5}{2}$ & 0 & $-\frac{1}{6}$ & $\frac{3}{2}$ & $-\frac{2}{3}$ & -1 & $S_{8}$ \\
\hline \hline$T_{1} / T_{5} *$ & $(1 ; 1,1)$ & 0 & $*$ & $\frac{5}{2}$ & $-\frac{1}{2}$ & $-\frac{2}{3}$ & $\frac{1}{2}$ & $\frac{1}{3}$ & 0 & $S_{1}$ \\
& $(1 ; 1,1)$ & 0 & $*$ & $-\frac{5}{2}$ & $\frac{1}{2}$ & $-\frac{2}{3}$ & $-\frac{1}{2}$ & $\frac{1}{3}$ & 0 & $S_{2}$ \\
& $2 \times(1 ; 1,1)$ & 0 & $*$ & $\frac{5}{2}$ & $\frac{1}{2}$ & $\frac{1}{3}$ & $\frac{1}{2}$ & $\frac{1}{3}$ & 0 & $S_{3,4}$ \\
& $2 \times(1 ; 1,1)$ & 0 & $*$ & $-\frac{5}{2}$ & $-\frac{1}{2}$ & $\frac{1}{3}$ & $-\frac{1}{2}$ & $\frac{1}{3}$ & 0 & $S_{5,6}$ \\
& $(1 ; \overline{\mathbf{3}}, 1)$ & 1 & $*$ & $\frac{5}{2}$ & 0 & $-\frac{1}{6}$ & $-\frac{1}{2}$ & $\frac{1}{3}$ & 0 & \\
& $(1 ; 1,1)$ & 1 & $*$ & $\frac{5}{2}$ & 0 & $-\frac{1}{6}$ & $-\frac{1}{2}$ & $-\frac{2}{3}$ & 1 & $S_{7}$ \\
\hline \hline$T_{3}$ & $(1 ; \mathbf{3}, 1)$ & $*$ & $-\frac{1}{3}$ & $\frac{5}{2}$ & $-\frac{1}{2}$ & 0 & $\frac{1}{2}$ & 0 & 1 & \\
& $(1 ; \overline{\mathbf{3}}, 1)$ & $*$ & $-\frac{1}{3}$ & $-\frac{5}{2}$ & $\frac{1}{2}$ & 0 & $-\frac{1}{2}$ & 0 & -1 & \\
\hline
\end{tabular}

Table A.8: Local states from the sectors $T_{1} / T_{5}$ and $T_{3}$ at $n_{2}=0 . T_{1} / T_{5} *$ denotes oscillator states. $S_{1}, S_{2}, S_{7}$ and $(1 ; \overline{\mathbf{3}}, 1)$ from that sector have oscillator numbers $\widetilde{N}^{*}=(1,0,0), S_{3}$ and $S_{5}$ have $\widetilde{N}^{*}=(0,1,0), S_{4}$ and $S_{6}$ have $\widetilde{N}^{*}=(2,0,0)$. 


\begin{tabular}{|c||c|c|c||c|c|c|c|c|c|c|c||c|}
\hline Sector & $n_{2}=1$ & $n_{3}$ & $q_{\gamma}$ & $t_{6}^{1}$ & $t_{7}$ & $t_{8}$ & $t_{1}$ & $t_{2}$ & $t_{3}$ & $t_{4}$ & $t_{5}$ & \\
\hline \hline$T_{1} / T_{5}$ & $(\mathbf{2}, 1 ; 1,1)$ & 0 & $*$ & 0 & 1 & -1 & $-\frac{1}{2}$ & $-\frac{1}{6}$ & 0 & $-\frac{5}{12}$ & $\frac{1}{4}$ & $M_{1}$ \\
& $(1,1 ; 1,1)$ & 0 & $*$ & 10 & 1 & -1 & $\frac{1}{2}$ & $-\frac{1}{6}$ & -1 & $-\frac{5}{12}$ & $\frac{1}{4}$ & $S_{1}^{-}$ \\
& $(1,1 ; 1,1)$ & 0 & $*$ & -10 & 1 & -1 & $\frac{1}{2}$ & $-\frac{1}{6}$ & 1 & $-\frac{5}{12}$ & $\frac{1}{4}$ & $S_{1}^{+}$ \\
& $(\mathbf{2}, 1 ; 1,1)$ & 1 & $*$ & 0 & -1 & 1 & 0 & $\frac{1}{3}$ & -1 & $\frac{1}{12}$ & $\frac{3}{4}$ & $M_{2}$ \\
& $(1,1 ; 1,1)$ & 1 & $*$ & 10 & -1 & 1 & $\frac{1}{2}$ & $-\frac{1}{6}$ & 1 & $\frac{1}{12}$ & $\frac{3}{4}$ & $S_{2}^{-}$ \\
& $(1,1 ; 1,1)$ & 1 & $*$ & -10 & -1 & 1 & 0 & $-\frac{2}{3}$ & 0 & $\frac{1}{12}$ & $\frac{3}{4}$ & $S_{2}^{+}$ \\
& $(\mathbf{2}, 1 ; 1,1)$ & 2 & $*$ & 0 & -1 & -1 & 0 & $\frac{1}{3}$ & 1 & $-\frac{5}{12}$ & $\frac{1}{4}$ & $M_{3}$ \\
& $(\mathbf{2}, 1 ; 1,1)$ & 2 & $*$ & 0 & 1 & 1 & 0 & $\frac{1}{3}$ & 1 & $\frac{1}{12}$ & $\frac{3}{4}$ & $M_{4}$ \\
& $(1,1 ; 1,1)$ & 2 & $*$ & 10 & -1 & -1 & 0 & $-\frac{2}{3}$ & 0 & $-\frac{5}{12}$ & $\frac{1}{4}$ & $S_{3}^{-}$ \\
& $(1,1 ; 1,1)$ & 2 & $*$ & -10 & -1 & -1 & $\frac{1}{2}$ & $-\frac{1}{6}$ & -1 & $-\frac{5}{12}$ & $\frac{1}{4}$ & $S_{3}^{+}$ \\
& $(1,1 ; 1,1)$ & 2 & $*$ & 10 & 1 & 1 & 0 & $-\frac{2}{3}$ & 0 & $\frac{1}{12}$ & $\frac{3}{4}$ & $S_{4}^{-}$ \\
& $(1,1 ; 1,1)$ & 2 & $*$ & -10 & 1 & 1 & $\frac{1}{2}$ & $-\frac{1}{6}$ & -1 & $\frac{1}{12}$ & $\frac{3}{4}$ & $S_{4}^{+}$ \\
\hline \hline \multirow{6}{*}{$T_{3}$} & $(1,1 ; 1,1)$ & $*$ & 0 & 10 & 1 & -1 & 0 & 0 & 2 & $\frac{1}{4}$ & $\frac{1}{4}$ & $S_{5}^{-}$ \\
& $(1,1 ; 1,1)$ & $*$ & 1 & 10 & 1 & -1 & 0 & 0 & 2 & $\frac{1}{4}$ & $\frac{1}{4}$ & $S_{5}^{\prime}$ \\
& $(1,1 ; 1,1)$ & $*$ & $\frac{1}{3}$ & -10 & -1 & 1 & 0 & 0 & -2 & $-\frac{1}{4}$ & $-\frac{1}{4}$ & $S_{5}^{+}$ \\
& $(1,1 ; 1,1)$ & $*$ & $\frac{1}{3}$ & 10 & 1 & -1 & $-\frac{1}{2}$ & $-\frac{1}{2}$ & -1 & $\frac{1}{4}$ & $\frac{1}{4}$ & $S_{6}^{-}$ \\
& $(1,1 ; 1,1)$ & $*$ & 0 & -10 & -1 & 1 & $\frac{1}{2}$ & $\frac{1}{2}$ & 1 & $-\frac{1}{4}$ & $-\frac{1}{4}$ & $S_{6}^{+}$ \\
& $(1,1 ; 1,1)$ & $*$ & 1 & -10 & -1 & 1 & $\frac{1}{2}$ & $\frac{1}{2}$ & 1 & $-\frac{1}{4}$ & $-\frac{1}{4}$ & $S_{6}^{+}$ \\
& $(1,1 ; 1,1)$ & $*$ & $-\frac{1}{3}$ & 10 & 1 & -1 & $\frac{1}{2}$ & $\frac{1}{2}$ & -1 & $\frac{1}{4}$ & $\frac{1}{4}$ & $S_{7}^{-}$ \\
& $(1,1 ; 1,1)$ & $*$ & $-\frac{1}{3}$ & -10 & -1 & 1 & $-\frac{1}{2}$ & $-\frac{1}{2}$ & 1 & $-\frac{1}{4}$ & $-\frac{1}{4}$ & $S_{7}^{+}$ \\
\hline
\end{tabular}

Table A.9: Local states from the sectors $T_{1} / T_{5}$ and $T_{3}$ at $n_{2}=1$. 


\section{B. Anomaly Polynomials}

In Section 4 , we checked that the irreducible terms in the anomaly polynomial cancel. The remaining piece explicitly reads

$$
\begin{aligned}
\mathrm{i}(2 \pi)^{3} I_{8}^{\mathrm{bulk}}=\frac{1}{16} & \left\{\left(\operatorname{tr} R^{2}\right)^{2}-\frac{1}{6}\left(\operatorname{tr} R^{2}\right)\left(\sum_{A} m_{A} \operatorname{tr} F_{A}^{2}+\sum_{u, v} m_{u v} F_{u} F_{v}\right)\right. \\
& \left.+4 \sum_{A, u, v} d_{A u v}\left(\operatorname{tr} F_{A}^{2}\right) F_{u} F_{v}+\frac{2}{3} \sum_{u, v, w, x} h_{u v w x} F_{u} F_{v} F_{w} F_{x}\right\},
\end{aligned}
$$

with coefficients

$$
\begin{aligned}
m_{A} & =\sum_{\mathbf{r}} s_{A}^{\mathbf{r}} v_{A}^{\mathbf{r}}-v_{A}^{(\text {adj })}, & m_{u v} & =\operatorname{tr}_{6}\left(t_{u} t_{v}\right)=\sum_{i} q_{u}^{i} q_{v}^{i} \\
d_{A u v} & =\sum_{\mathbf{r}} v_{A}^{\mathbf{r}} \sum_{k=1}^{s_{A}^{\mathbf{r}}} q_{u}^{k} q_{v}^{k}, & h_{u v w x} & =\operatorname{tr}_{6}\left(t_{u} t_{v} t_{w} t_{x}\right)=\sum_{i} q_{u}^{i} q_{v}^{i} q_{w}^{i} q_{x}^{i} .
\end{aligned}
$$

All sums are over hypermultiplets only; the vector multiplets only appear in the final term of $m_{A}$. In the sums, $i$ runs over all states, $\mathbf{r}$ over all representations of group $G_{A}$ and $k$ over all multiplets in representation $\mathbf{r} . q_{u}^{i}$ and $q_{u}^{k}$ are the charges of states and multiplets under $U(1)_{u}$, and $\operatorname{tr}_{6}$ denotes the trace of the $\mathrm{U}(1)$ generators, i.e. the sum over the charges of all fields. The integers $s_{A}^{\mathbf{r}}$ are the multiplicities of states transforming in that representation, and $v_{A}^{\mathbf{r}}$ is its quadratic index. Note that terms $\sim\left(\operatorname{tr} F_{A}^{2}\right)\left(\operatorname{tr} F_{B}^{2}\right)$ for two different non-Abelian factors $A, B$ add up to zero in our model. By explicit evaluation of these definitions in the basis $\hat{t}_{u}=t_{u} / \sqrt{2}\left|t_{u}\right|$ we find the results

$$
m_{A}=6(2,2,1), \quad m_{u v}=6\left(\beta_{u v}+\delta_{u v}\right)
$$

where $\beta_{u v}$ is given in Eq. (4.12). Furthermore

$$
\begin{aligned}
d_{\mathrm{SU}(6) u v} & =d_{\mathrm{SU}(3) u v}=2 d_{\mathrm{SO}(8) u v}=\frac{1}{2} \beta_{u v}, \\
h_{u v w x} & =\frac{3}{2|\sigma(u v w x)|}\left(\delta_{u v} \beta_{w x}+\text { perm. }\right),
\end{aligned}
$$

where $|\sigma(u v w x)|$ counts all possible distinct permutations of indices $u, v, w, x$ (and only these are included in the bracket).

Similarly, we calculate the local anomaly polynomial at a fixed point $f$ :

$$
i(2 \pi)^{3} I_{6}^{f}=-\frac{1}{48} \sum_{u} m_{u}^{f} F_{u} \operatorname{tr} R^{2}+\frac{1}{2} \sum_{A, u} d_{A u}^{f} F_{u} \operatorname{tr} F_{A}^{2}+\frac{1}{6} \sum_{u v w} h_{u v w}^{f} F_{u} F_{v} F_{w} .
$$


Here the coefficients are defined as follows:

$$
\begin{aligned}
m_{u}^{f} & =\operatorname{tr}_{f}\left(t_{u}^{f}\right)=\sum_{i} b^{i} q_{u}^{i}, & d_{A u}^{f}=\sum_{\mathbf{r}} v_{A}^{\mathrm{r}} \sum_{k=1}^{s_{A}^{\mathrm{r}}} b^{k} q_{u}^{k}, \\
h_{u v w}^{f} & =\operatorname{tr}_{f}\left(t_{u}^{f} t_{v}^{f} t_{w}^{f}\right)=\sum_{i} b^{i} q_{u}^{i} q_{v}^{i} q_{w}^{i} &
\end{aligned}
$$

All sums refer to the local spectrum at fixed point $f$, evaluated on left-handed fields. The local $\operatorname{trace} \operatorname{tr}_{f}$ contains an additional factor $b^{i}$, which is either one for localized states or $1 / 4$ for states which are induced by bulk fields; the same holds for $b^{k}$. We conveniently evaluate these expressions in a basis which consists of $\hat{t}_{\mathrm{an}}^{f}=t_{\mathrm{an}}^{f} / \sqrt{2}\left|t_{\mathrm{an}}^{f}\right|$, with $t_{\text {an }}^{f}$ from Table 4.1, and orthogonal generators, $\hat{t}_{1}^{f} \equiv \hat{t}_{\text {an }}^{f}, \hat{t}_{\text {an }}^{f} \cdot \hat{t}_{u}^{f}=0(u>1)$. Then the only non-vanishing terms are

$$
\operatorname{tr}_{0} \hat{t}_{\mathrm{an}}^{0}=2 \sqrt{37}, \quad \operatorname{tr}_{1} \hat{t}_{\mathrm{an}}^{1}=2 \sqrt{10}
$$

and

$$
\begin{aligned}
d_{\mathrm{SU}(5) \text { an }}^{0} & =d_{\mathrm{SU}(3) \text { an }}^{0}=2 d_{\mathrm{SO}(8) \text { an }}^{0}=2 \operatorname{tr}_{0} \hat{t}_{\mathrm{an}}^{0}\left(\hat{t}_{u}^{0}\right)^{2}=\frac{2}{3} \operatorname{tr}_{0}\left(\hat{t}_{\mathrm{an}}^{0}\right)^{3}=\frac{1}{12} \operatorname{tr}_{0} \hat{t}_{\mathrm{an}}^{0}, \\
d_{\mathrm{SU}(2) \text { an }}^{1} & =d_{\mathrm{SU}(4) \text { an }}^{1}=d_{\mathrm{SU}(2)^{\prime} \text { an }}^{1}=d_{\mathrm{SU}(4)^{\prime} \text { an }}^{1} \\
& =2 \operatorname{tr}_{0} \hat{t}_{\mathrm{an}}^{1}\left(\hat{t}_{u}^{1}\right)^{2}=\frac{2}{3} \operatorname{tr}_{0}\left(\hat{t}_{\mathrm{an}}^{1}\right)^{3}=\frac{1}{12} \operatorname{tr}_{0} \hat{t}_{\mathrm{an}}^{1} .
\end{aligned}
$$

This shows explicitly that both anomaly polynomials factorize in the required way, Eq. (4.5), i.e. the Green-Schwarz universality relations with levels $\alpha_{\mathrm{SO}(N)}=1$ and $\alpha_{\mathrm{SU}(N)}=2$ are fulfilled,

$$
\frac{1}{48} \operatorname{tr}_{f} \hat{t}_{\mathrm{an}}^{f}=\frac{1}{6} \operatorname{tr}_{f}\left(\hat{t}_{\mathrm{an}}^{f}\right)^{3}=\frac{1}{2} \operatorname{tr}_{f} \hat{t}_{\mathrm{an}}^{f}\left(\hat{t}_{u}^{f}\right)^{2}=\frac{1}{2 \alpha_{A}} d_{A \text { an }}^{f} .
$$




\section{References}

[1] H. Georgi and S. L. Glashow, Phys. Rev. Lett. 32 (1974), 438.

[2] H. Georgi, in: Particles and Fields 1974, ed. C. E. Carlson (AIP, NY, 1975) p. 575.

[3] H. Fritzsch and P. Minkowski, Ann. Phys. 93 (1975), 193.

[4] D. J. Gross, J. A. Harvey, E. J. Martinec and R. Rohm, Phys. Rev. Lett. 54 (1985), 502.

[5] D. J. Gross, J. A. Harvey, E. J. Martinec and R. Rohm, Nucl. Phys. B256 (1985), 253.

[6] L. J. Dixon, J. A. Harvey, C. Vafa and E. Witten, Nucl. Phys. B261 (1985), 678.

[7] L. J. Dixon, J. A. Harvey, C. Vafa and E. Witten, Nucl. Phys. B274 (1986), 285.

[8] L. E. Ibáñez, H. P. Nilles and F. Quevedo, Phys. Lett. B187 (1987), 25.

[9] L. E. Ibáñez, J. E. Kim, H. P. Nilles and F. Quevedo, Phys. Lett. B191 (1987), 282.

[10] For reviews and references, see D. Bailin and A. Love, Phys. Rept. 315 (1999) 285;

M. K. Gaillard and B. D. Nelson, [hep-th/0703227].

[11] T. Kobayashi, S. Raby and R.-J. Zhang, Phys. Lett. B593 (2004), 262

[hep-ph/0403065].

[12] S. Förste, H. P. Nilles, P. K. S. Vaudrevange and A. Wingerter, Phys. Rev. D70 (2004), 106008 [hep-th/0406208].

[13] T. Kobayashi, S. Raby and R.-J. Zhang, Nucl. Phys. B704 (2005), 3 [hep-ph/0409098].

[14] A. Hebecker and M. Trapletti, Nucl. Phys. B 713 (2005) 173 [hep-th/0411131].

[15] W. Buchmüller, K. Hamaguchi, O. Lebedev and M. Ratz, Nucl. Phys. B712 (2005), 139 [hep-ph/0412318].

[16] W. Buchmüller, K. Hamaguchi, O. Lebedev and M. Ratz, [hep-ph/0512326] .

[17] W. Buchmüller, K. Hamaguchi, O. Lebedev and M. Ratz, Phys. Rev. Lett. 96 (2006), 121602 [hep-ph/0511035].

[18] W. Buchmüller, K. Hamaguchi, O. Lebedev and M. Ratz, Nucl. Phys. B 785, 149 (2007) [hep-th/0606187].

[19] O. Lebedev, H. P. Nilles, S. Raby, S. Ramos-Sanchez, M. Ratz, P. K. S. Vaudrevange and A. Wingerter, Phys. Lett. B 645 (2007) 88 [hep-th/0611095].

[20] M. B. Green and J. H. Schwarz, Phys. Lett. B 149 (1984) 117. 
[21] J. Erler, J. Math. Phys. 35 (1994) 1819 [hep-th/9304104].

[22] H. M. Lee, H. P. Nilles and M. Zucker, Nucl. Phys. B 680 (2004) 177 [hep-th/0309195].

[23] For a review, see A. Font and S. Theisen, "Introduction To String Compactification," Lect. Notes Phys. 668 (2005) 101.

[24] H. Nishino and E. Sezgin, Nucl. Phys. B 278 (1986) 353.

[25] N. Arkani-Hamed, T. Gregoire and J. G. Wacker, JHEP 0203 (2002) 055 [hep-th/0101233].

[26] For a review and references, see C. A. Scrucca and M. Serone, Int. J. Mod. Phys. A 19 (2004) 2579 [hep-th/0403163].

[27] T. Asaka, W. Buchmüller and L. Covi, Nucl. Phys. B 648 (2003) 231 [hep-ph/0209144].

[28] G. von Gersdorff and M. Quiros, Phys. Rev. D 68 (2003) 105002 [hep-th/0305024].

[29] G. von Gersdorff, JHEP 0703 (2007) 083 [hep-th/0612212].

[30] R. Stora, in Progress in Gauge Field Theory, eds. G. 't Hooft et al., Plenum Press 1984, pp 373-401;

B. Zumino, in Relativity, groups and topology II, eds. B. DeWitt and R. Stora, Elsevier, 1984.

[31] A. Font, L. E. Ibanez, H. P. Nilles and F. Quevedo, Nucl. Phys. B 307 (1988) 109 [Erratum ibid. B 310 (1988) 764].

[32] S. G. Nibbelink, M. Trapletti and M. Walter, JHEP 0703 (2007) 035 [hep-th/0701227].

[33] W. Buchmüller, K. Hamaguchi, O. Lebedev, S. Ramos-Sanchez and M. Ratz, Phys. Rev. Lett. 99 (2007) 021601 [hep-ph/0703078].

[34] G. Cleaver, M. Cvetic, J. R. Espinosa, L. L. Everett and P. Langacker, Nucl. Phys. B 525 (1998) 3 [arXiv:hep-th/9711178]. 